Revue des patrimoines

$31 \mid 2017$

Patrimoines de la santé : essais de définition - enjeux de conservation

\title{
Valoriser le patrimoine climatique : la reconversion des sanatoriums de cure antituberculeuse
}

Interpreting the climate heritage; the conversion of tuberculosis sanatoriums

\section{Philippe Grandvoinnet}

\section{(2) OpenEdition}

\section{Journals}

Édition électronique

URL : http://journals.openedition.org/insitu/14173

DOI : $10.4000 /$ insitu. 14173

ISSN : 1630-7305

Éditeur

Ministère de la Culture

Référence électronique

Philippe Grandvoinnet, « Valoriser le patrimoine climatique : la reconversion des sanatoriums de cure antituberculeuse », In Situ [En ligne], 31 | 2017, mis en ligne le 23 février 2017, consulté le 09 octobre 2020. URL : http://journals.openedition.org/insitu/14173; DOI : https://doi.org/10.4000/insitu.14173

Ce document a été généré automatiquement le 9 octobre 2020.

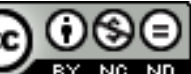

In Situ Revues des patrimoines est mis à disposition selon les termes de la licence Creative Commons Attribution - Pas d'Utilisation Commerciale - Pas de Modification 4.0 International. 


\title{
Valoriser le patrimoine climatique : la reconversion des sanatoriums de cure antituberculeuse
}

\author{
Interpreting the climate heritage; the conversion of tuberculosis sanatoriums
}

\author{
Philippe Grandvoinnet
}

1 Soixante années de lutte intensive contre la tuberculose ${ }^{1}$ ont laissé en Europe un patrimoine bâti dont on commence aujourd'hui seulement à mesurer l'ampleur, les qualités, mais aussi la fragilité. Rien qu'en France, environ trois cent cinquante sanatoriums de cure (établissements publics et privés confondus) ont été construits à neuf ou aménagés dans des bâtiments existants entre 1900 et $1960^{2}$. " Maladie de l'élément aérien ${ }^{3}$ ", la tuberculose a ceci de paradoxal que l'air, reconnu comme le principal vecteur de la maladie lorsqu'il est vicié et porteur de germes, fut considéré jusqu'au début des années 1950 lorsqu'il est sain comme le premier adjuvant de la cure. Il reste de cette aventure sanitaire sans précédent des édifices parfaitement identifiables en raison de leurs caractères typologiques: une orientation solaire affirmée qui oppose une façade sud thérapeutique à une façade nord dévolue aux services, des corps de bâtiment peu épais conçus pour faciliter la pénétration de l'air et de la lumière, un développement longitudinal accentué par la présence de galeries de cure. Ces caractères constituent la marque de fabrique la plus visible du programme sanatorial et distinguent les sanatoriums des autres types hospitaliers issus de la spécialisation des établissements d'hygiène et de santé à partir du milieu du XIX ${ }^{\mathrm{e}}$ siècle.

2 La reconversion des sanatoriums de cure a été engagée à la fin des années 1960 lorsque les thérapies antibiotiques ont privé les établissements de leurs patients tuberculeux. D'abord administrative, la reconversion des sanatoriums n'a véritablement eu un impact sur leur architecture qu'à partir des années 1990, lorsque leur vocation médicale a été remise en question. Pour les stations climatiques de cure, spécialisées dans l'activité médicale depuis deux générations au moins, ce processus a conduit à une mutation profonde du tissu économique et social. A quelques rares exceptions près, l'enjeu architectural et urbain n'a pas été perçu immédiatement: en France, les 
démolitions et les rénovations lourdes ont conduit à la perte d'une part importante de l'histoire et $d u$ patrimoine climatiques $d u x^{e}$ siècle. Alors qu'elle était menacée, l'architecture climatique est peu à peu apparue comme un levier économique potentiel pour les territoires, mais aussi comme un marqueur culturel et d'identité locale. Son recyclage, sa transformation et sa restauration sont à présent un enjeu partagé par les propriétaires et gestionnaires d'établissements, la société civile, les collectivités et les services de l'État en charge de l'architecture et du patrimoine.

\section{Architecture thérapeutique}

3 La force d'évocation des anciens sanatoriums de cure, même à l'état d'abandon, provient en grande partie de la clarté de leurs dispositions architecturales et techniques. Pour qui sait les lire, la façade, le plan et les aménagements intérieurs de ces établissements forment les éléments d'une mécanique hygiénique complexe dont l'objectif unique était d'améliorer le "rendement thérapeutique » de la cure en plaçant les malades dans des conditions propices à leur guérison. Cette lisibilité s'exprime essentiellement dans l'articulation du plan et des élévations: les façades d'un sanatorium de cure - à commencer par la façade sud, active d'un point de vue thérapeutique - permettent de « déceler au dehors les nécessités vraies du plan ${ }^{4}$ » et de rendre perceptibles la structure et l'organisation globale de l'édifice. Cette clarté fonctionnelle fait bien entendu écho à la transparence littérale des corps de bâtiments dont l'insolation et la ventilation intensives garantissaient la parfaite asepsie.

4 L'identité architecturale d'un sanatorium repose donc sur sa qualité de programme médical et social spécifique. Ce programme est précis, scientifiquement déterminé, mais aussi sujet à de fréquentes variations dans le temps, à l'image des thérapies employées dans le traitement de la tuberculose pulmonaire. Pour ces raisons, les sanatoriums ne sont pas figés dans des dispositifs spatiaux reproductibles, chaque projet est une nouvelle interprétation du programme en fonction des conceptions thérapeutiques du moment :

L'architecte, pour les bâtir, n'est plus tenu à aucune considération sentimentale ou littéraire ; il n'a rien à exprimer : ses intentions doivent être pures, comme celles du forgeron qui fabrique un marteau ou du lamineur qui étire un fer profilé ; la seule

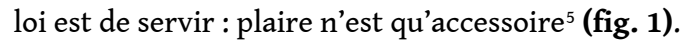




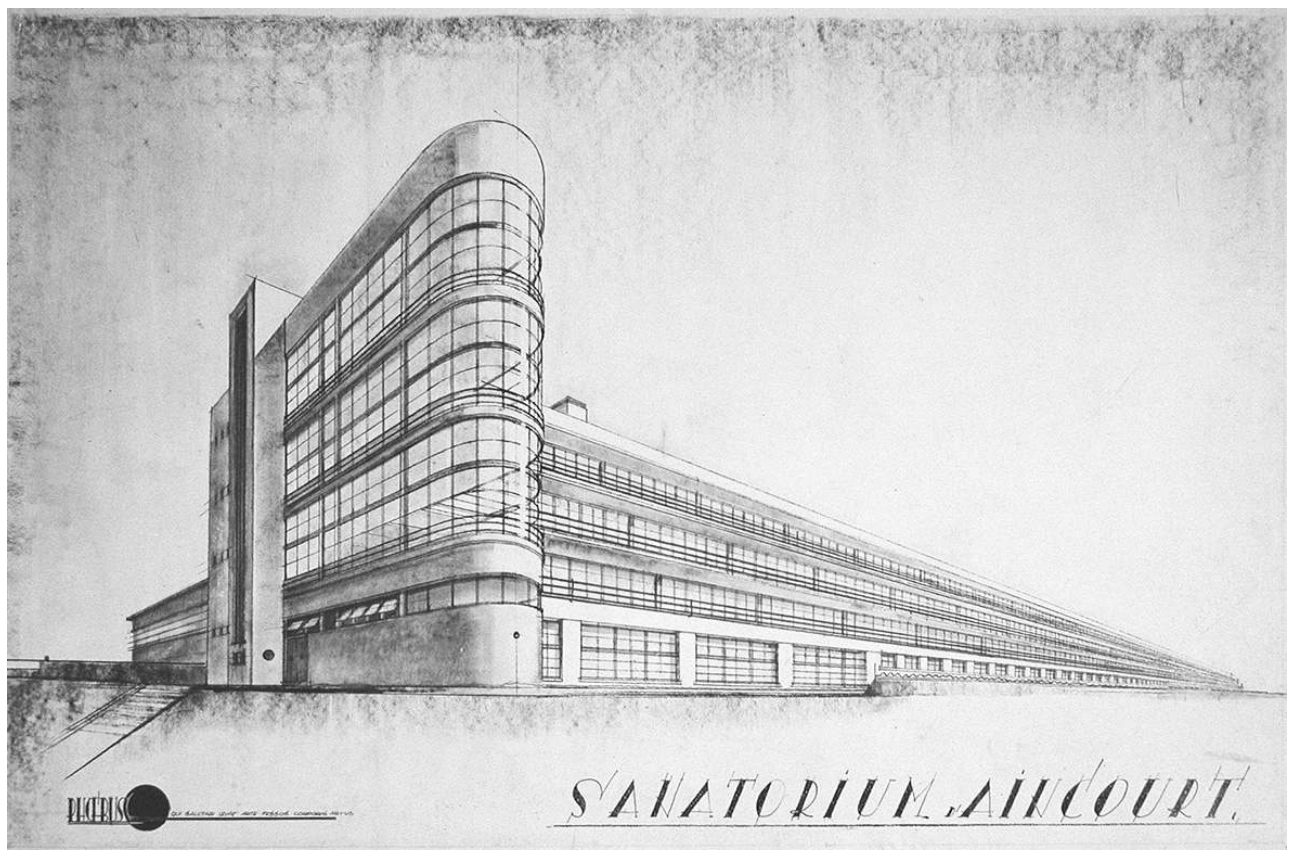

Projet pour le sanatorium populaire à Aincourt (Val-d'Oise, Georges-Henri Pingusson architecte, 1929). L'Architecture d'aujourd'hui, 1934

5 Certains sanatoriums, tels les établissements de Görbersdorf (Silésie, auj. Sokolowsko, Pologne, 1862), de Hauteville (France, Ain, 1900) ou de Leysin (Suisse, années 1910) ont marqué l'histoire de la lutte antituberculeuse par les méthodes thérapeutiques novatrices qui y ont été développées. On constate toutefois que les lieux de l'innovation médicale ont rarement été ceux de l'innovation architecturale ou technique: expérimentées dans les établissements existants, les améliorations apportées aux méthodes de cure n'ont été pleinement mises en œuvre que dans des projets postérieurs à l'architecture plus audacieuse. Ce décalage s'explique par l'évolution rapide des techniques médicales, tandis que les formes architecturales restent souvent fidèles à la conception sanatoriale classique, forgée au XIX ${ }^{e}$ siècle et éprouvée par la pratique. Les anciens établissements de tuberculeux sont donc les héritiers d'une double histoire, médicale et architecturale, qu'il importe de considérer pour replacer chaque projet dans la production de l'époque.

Paradoxalement, les sanatoriums ne ressemblent pas à des hôpitaux et c'est un choix délibéré : afin de rendre la vie en sanatorium plus supportable - les malades pouvaient en effet y séjourner plusieurs années - les établissements ont été volontairement débarrassés de références hospitalières. Les préoccupations des médecins et celles des architectes se rejoignaient dans le même souci du bien-être des tuberculeux, pour lesquels on s'attachait à créer un cadre de vie confortable. Outre les enduits colorés et les intérieurs peints de teintes vives, les matériaux de revêtement participaient aussi au décor: s'ils étaient choisis avant tout pour leur solidité et leur résistance à la désinfection, le grès cérame, le granito ou le linoléum offraient également une variété infinie de teintes et de motifs décoratifs. Ces éléments constituaient l'essentiel de la décoration intérieure des établissements qui, loin d'exprimer une asepsie austère, se paraient d'une sorte de luxe discret. L'abandon progressif, à partir des années 1960, des méthodes classiques de la cure antituberculeuse, a toutefois conduit à la réalisation 
d'aménagements successifs qui ont dilué cette spécificité sanatoriale dans une sorte de banal hospitalier fait de blanc et d'espaces polyvalents sans identité. Les contraintes réglementaires ont également surchargé ces bâtiments de couches successives d'emplâtres (faux planchers, faux plafonds et autres dispositifs pour dissimuler les équipements techniques), jusqu'à brouiller totalement la lecture que l'on pouvait avoir de ces espaces. Ces aménagements ne sauraient mieux détourner les sanatoriums du principe de "sincérité architecturale ${ }^{6}$ » qui leur confère une indéniable qualité spatiale et procure à leurs usagers une satisfaction autant sensible qu'intellectuelle. Enfin, le sanatorium est porteur de valeurs sanitaires et sociales fondées sur le lien qui unit l'architecture et son environnement : transparent, il entretient une relation consciente au site qui l'accueille; démocratique, il attribue à chaque occupant une portion identique de soleil et de paysage.

\section{L'évolution des types architecturaux}

7 Les formes architecturales des sanatoriums ont considérablement évolué entre la fin du $\mathrm{XIX}^{\mathrm{e}}$ et le milieu du $\mathrm{xx}^{\mathrm{e}}$ siècle. Le modèle initial est développé en Allemagne dans les années 1880-1890: il se compose d'un bâtiment d'hospitalisation unique ou de pavillons alignés orientés au sud. Les galeries de cure sont alors dispersées dans le parc de l'établissement ou adossées au rez-de-chaussée des bâtiments. C'est le modèle des premiers sanatoriums populaires allemands (Ruppertshain, dans le Taunus, 1892) et suisses (Wald, canton de Zürich, 1895), puis français (Hauteville, 1900) et italiens (Pineta di Sortenna, en Lombardie, 1902). Il reste en vigueur jusqu'à la Première Guerre mondiale et l'on en trouve des réminiscences dans un grand nombre d'établissements de l'entre-deux-guerres. Les années 1910 et 1920 sont marquées par des expériences contrastées résultant d'injonctions contradictoires qui préconisaient de concentrer les services dans un bâtiment unique afin d'en simplifier la gestion ou au contraire de faire éclater le plan pour offrir aux malades le maximum d'air et de lumière. Le modèle pavillonnaire connaît un certain succès aux États-Unis (sanatorium Trudeau, lac Saranac, New York, 1885) et en Angleterre (village-sanatorium de Papworth, Cambridge, 1911) mais ne dépasse pas le stade de l'expérimentation en France (villagessanatoriums de Plougonven, Finistère, 1923, et de Praz-Coutant en Haute-Savoie, 1926). À partir de 1900 dans les pays germaniques, de 1920 en France, en Italie et dans le sud de l'Europe, les grands sanatoriums publics adoptent un plan classique en $\mathrm{T}$ inversé formé d'une longue aile d'hospitalisation exposée au sud et d'une aile transversale de services orientée au nord. Alors que la chambre individuelle représente un standard dans les sanatoriums privés, les malades sont logés en dortoirs de trois à huit lits dans la plupart des sanatoriums populaires. Cela se traduit dans leur architecture par des modules de façade plus larges et des compositions plus étalées du fait de la grande capacité de ces établissements qui peuvent atteindre cinq cents lits. Les meilleurs exemples de ces dispositions sont les grands sanatoriums populaires français des années 1930, de plaine (Villiers-sur-Marne dans le Val-de-Marne, La Musse, dans l'Eure, Le Petit-Arbois, dans les Bouches-du-Rhône, Felleries-Liessies, dans le Nord) ou de montagne (Les Petites-Roches, Isère) avec toutefois de nombreuses variantes : ailes asymétriques, bâtiments étalés ou en hauteur, position des galeries de cure, protections contre les intempéries selon la nature du climat local. Dérogent à ce schéma les dispositions à gradins, très en vogue au début des années 1930 (Waiblingen, près de Stuttgart, en Allemagne, Aincourt dans le Val-d'Oise, Guébriant en Haute-Savoie ou 
Bodiffé dans les Côtes-d'Armor en France), certains hôpitaux-sanatoriums et sanatoriums suburbains, qui se plient plus volontiers aux modèles hospitaliers, ainsi que les établissements de la dernière génération, conçus peu avant la Seconde Guerre mondiale et jusqu'au milieu des années 1950 pour dispenser de nouvelles méthodes thérapeutiques, chirurgicales notamment. Si l'air marin ou le soleil de haute montagne étaient particulièrement indiqués dans le traitement des tuberculoses extrapulmonaires (scrofule, tuberculose ganglionnaire, etc.), le traitement de la tuberculose pulmonaire se pratiquait sous tous les climats, à l'exception des zones humides (propices au développement du bacille de Koch), ventées (les tuberculeux étant sensibles aux courants d'air) ou exposées à la pollution atmosphérique (source d'irritation pulmonaire). Ces contraintes climatiques ont conduit à repousser les sanatoriums hors des zones habitées, le plus souvent à la campagne ou dans des zones boisées, jusqu'à des altitudes de 1350 à 1500 mètres (Briançon, dans les Hautes-Alpes, Davos, dans les Grisons), voire plus haut (le sanatorium d'Odeillo, dans les Pyrénées, est situé à 1850 mètres d'altitude). Si cet isolement garantissait à l'origine le calme, l'air pur et l'ensoleillement, il peut aujourd'hui constituer un obstacle à la réhabilitation des anciens établissements lorsqu'ils sont trop éloignés des agglomérations ou mal reliés aux grandes infrastructures de transports.

\section{Stratégies de projet}

8 Si l'architecture des sanatoriums répond à des critères typologiques définis, elle ne peut pas pour autant être réduite à des modèles-types (à l'exception de quelques productions locales ou nationales, italienne par exemple ${ }^{7}$ ). Les sanatoriums se caractérisent au contraire par la variété des formes, matériaux et dispositions retenus par leurs concepteurs. La diversité du corpus sanatorial justifie l'intérêt que leur portent aujourd'hui les historiens, les conservateurs du patrimoine, les architectes et les propriétaires de ces établissements lorsqu'ils envisagent de faire évoluer leur utilisation.

En France, la première vague de reconversion des sanatoriums a porté sur la simple conversion administrative des lits de tuberculeux en lits conventionnés pour d'autres types de pathologies. Il s'agissait pour les gestionnaires de choisir l'un des trois débouchés envisagés au début des années 1970 par l'administration : perpétuer la cure sanatoriale de la tuberculose (pour un nombre restreint de lits), engager une reconversion dans la cure climatique des bronchopathies ou positionner l'établissement dans les traitements de rééducation fonctionnelle ${ }^{8}$. On s'est alors contenté de mises aux normes réglementaires, essentiellement techniques, qui ont peu affecté l'architecture des établissements. Cette phase n'a duré qu'un temps et la question de leur reconversion architecturale s'est posée dans les années 1990 en raison du vieillissement des bâtiments et de leurs installations, de l'évolution des pratiques médicales et de la volonté d'optimiser l'offre de soins en rapprochant les établissements des centres urbains. Beaucoup de sanatoriums ont alors été démolis ou ont fait l'objet de restructurations lourdes qui ont conduit à la perte de leur cohérence d'origine ou d'une part importante de leur substance bâtie, dans le déni complet de leur spécificité climatique. Ces destructions s'expliquent par l'absence d'inventaire méthodique de ce qui n'était pas encore identifié comme un patrimoine à préserver. En l'absence de vision globale et partagée de cette production architecturale complexe, il 
était en effet difficile de fonder une méthode de projet susceptible d'interroger les potentialités de chaque bâtiment selon des critères historiques, techniques, architecturaux et paysagers qui lui sont propres'.

10 La création, en 1999, du label « Patrimoine du xx ${ }^{e}$ siècle » par le ministère de la Culture et de la Communication a contribué à faire évoluer le regard porté sur ces bâtiments. La vingtaine de sanatoriums labellisés depuis a permis de mettre en lumière les qualités architecturales et paysagères de ces réalisations. Cette reconnaissance a pu orienter les choix en matière de réhabilitation mais les projets restent marqués par des programmes parfois inadaptés à la structure même des édifices. Quelle que soit la stratégie adoptée (évolution progressive de l'activité médicale ou reconversion lourde), c'est un leurre de croire que des bâtiments à ce point marqués par leur identité typologique pourront supporter tous types d'usages. Construits sur la base de prescriptions techniques, sanitaires et sociales complexes, les sanatoriums ne sont pas moins exigeants lorsqu'il s'agit de les adapter à une nouvelle activité. Leur typologie, leur implantation et leur configuration spatiale limitent nécessairement le champ des possibles lorsqu'il faut définir les conditions de leur recyclage : le long développement des bâtiments, leur structuration en cellules identiques ou encore la dichotomie nette entre la façade sud et les espaces de service doivent orienter les choix en amont de la programmation, dès l'étude de faisabilité. La valeur ajoutée d'un projet de réhabilitation - qui justifie la conservation du bâtiment plutôt que sa démolition dépend plus de la capacité du maître d'ouvrage à adapter son programme aux spécificités du bâti que de l'habileté de l'architecte à plier le bâtiment aux exigences d'un programme inadapté. Créer les conditions d'une relation dialectique entre le bâtiment et son nouvel usage apparaît ici comme le véritable enjeu.

11 Plusieurs questions se posent alors : comment préserver les qualités spatiales originales d'un ancien sanatorium tout en admettant un changement d'usage? Comment préserver le lien fonctionnel qui unit le bâtiment à son environnement? À titre d'exemple, les questions relatives au confort thermique et à la gestion du climat intérieur doivent être posées en termes spécifiques : alors que la perméabilité à l'air des sanatoriums était un gage d'excellence sanitaire (dans le contexte particulier de la cure d'air), l'étanchéité des bâtiments à l'air est aujourd'hui un enjeu technique majeur, même lorsqu'il s'agit de réhabilitation. Ces contradictions devront pourtant être dépassées dans le cadre du projet architectural de transformation de l'existant. Les projets peuvent pour cela s'appuyer sur les qualités initiales du bâti : en plaçant le malade à l'interface de l'environnement naturel et du climat contrôlé, les sanatoriums répondent d'une certaine façon aux préoccupations actuelles des concepteurs et des usagers. Les qualités climatiques (orientation, protections solaires, enveloppe, inertie) des anciens sanatoriums de cure peuvent ainsi fonder une stratégie de projet de réhabilitation.

\section{La restauration des icônes}

12 Programme phare de l'architecture du $\mathrm{xx}^{\mathrm{e}}$ siècle, le sanatorium s'illustre par des réalisations d'exception qui ont acquis un statut d'«icônes du modernisme »: le sanatorium néerlandais Zonnestraal - «rayon de soleil» - (Jan Duiker architecte, Jan Gerko Wibenga ingénieur, 1926-1928), à Hilversum fait ainsi partie des réalisations emblématiques de l'avant-garde architecturale des années $1920^{10}$ : l'innovation 
architecturale et technique reposait ici sur la nouveauté du programme sanitaire. Les bâtiments ont fait l'objet d'une restauration méticuleuse menée par des praticiens expérimentés qui ont pris le temps d'élaborer un appareil critique, historique et technique ad hoc. Les architectes Hubert-Jan Henket et Wessel de Jonge ont consacré plus de dix ans à cette restauration qui, outre les qualités techniques du résultat, a l'immense mérite de définir un horizon d'excellence vers lequel toute intervention peut tendre (fig. 2). Achevée en 2000, cette restauration pose toutefois un certain nombre de questions: elle rappelle d'abord la nature transitoire de l'architecture sanatoriale et la difficulté de conserver dans de bonnes conditions une structure conçue pour ne durer qu'un temps ${ }^{11}$; elle abolit également les contraintes économiques courantes puisque l'opération a largement dépassé le coût d'un bâtiment neuf: l'économie du projet repose ici sur une occupation tertiaire haut de gamme (essentiellement des activités médicales, ainsi qu'un centre de congrès) liée à l'attractivité du site (un domaine boisé de cent vingt hectares situé à proximité du centre d'Hilversum). Si une opération de ce type est hors de portée de la plupart des anciens établissements de cure (pour des raisons autant économiques que d'opportunité), elle offre un cadre méthodologique exemplaire dont peut s'inspirer tout projet de réhabilitation ou de restauration ${ }^{12}$.

Figure 2

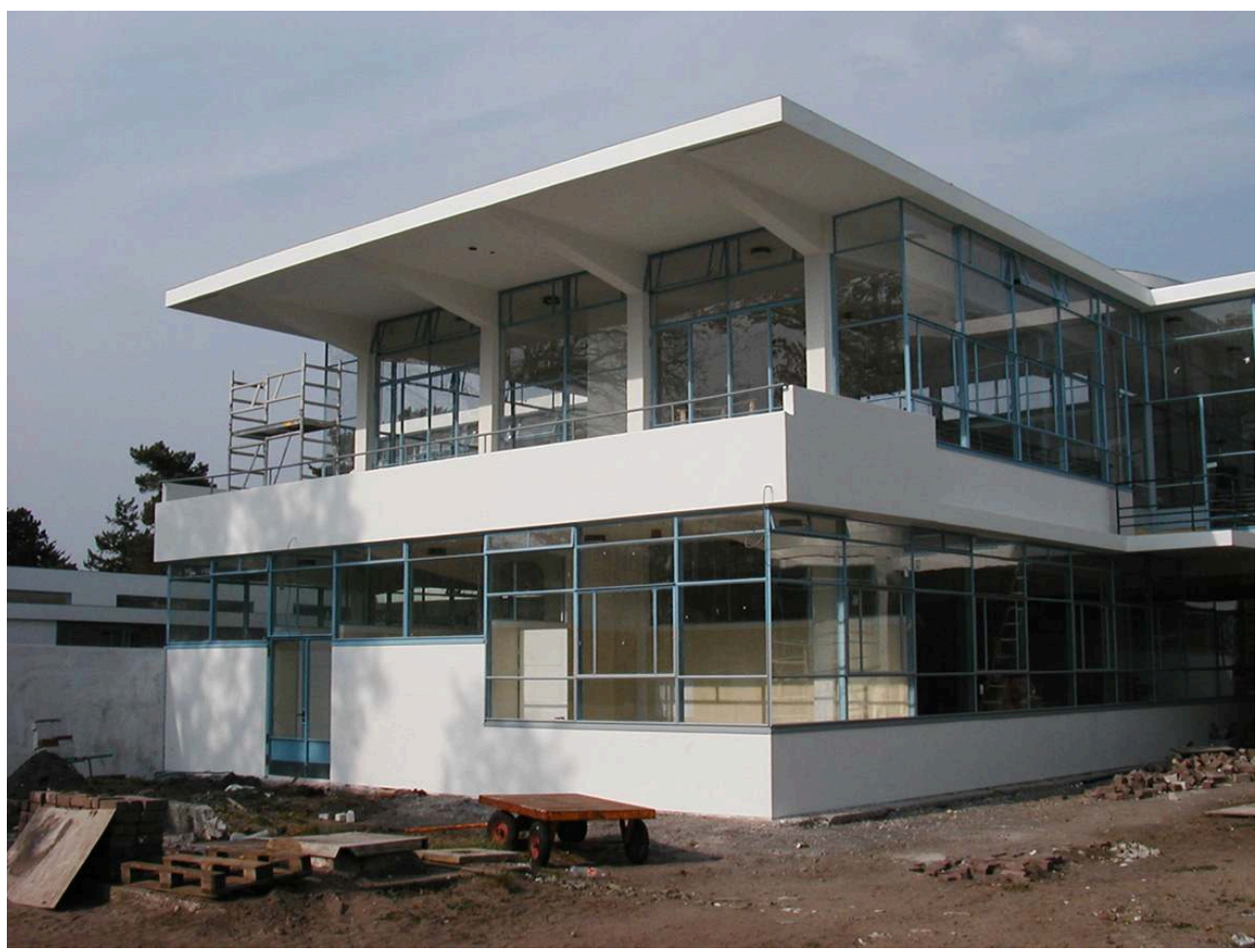

Sanatorium Zonnestraal (Hilversum : Pays-Bas, Jan Duiker architecte, 1928) : le bâtiment central en cours de restauration.

Phot. Grandvoinnet, Ph., 2003. (c) Philippe Grandvoinnet.

Zonnestraal est l'exemple sans doute le plus abouti d'une série de restaurations conduites dans les années 1990-2000 sur d'anciens sanatoriums d'intérêt majeur en Europe, tels les établissements Bella Lui, Schatzalp (fig. 3) et Clavadel (fig. 4) en Suisse, le sanatorium de Paimio en Finlande ou les sanatoriums de Beelitz en Allemagne. Ces 
établissements ont en commun d'avoir conservé sans interruption une activité de type médical ou hôtelier. Ils ont toutefois fait l'objet d'approches différentes en matière de protection et de valorisation du patrimoine architectural.

Figure 3

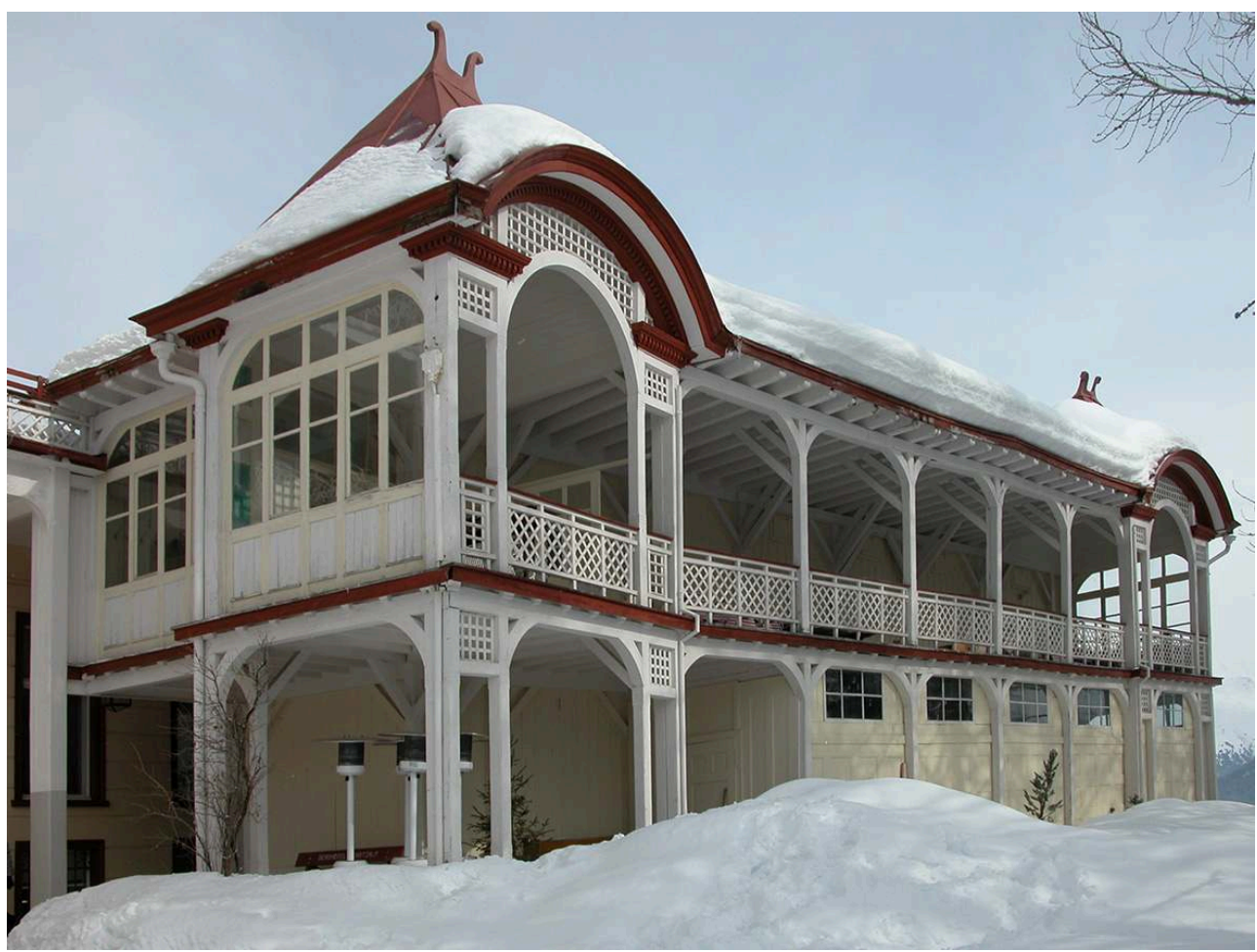

Sanatorium Schatzalp (Davos : Suisse, Pfleghardt et Häfeli architectes, 1900) : galerie de cure restaurée.

Phot. Grandvoinnet, Ph., 2003. (c) Philippe Grandvoinnet. 
Figure 4

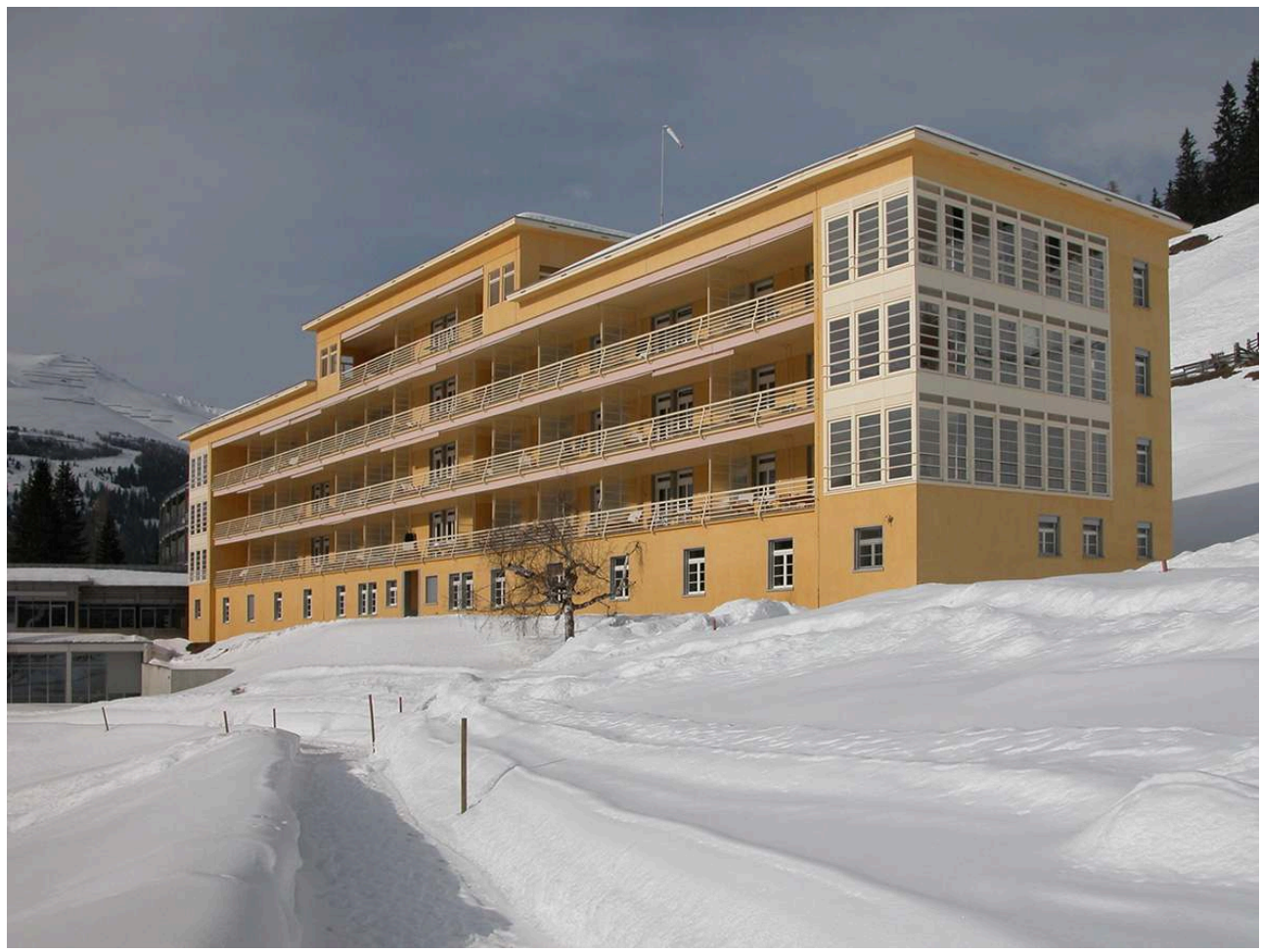

Sanatorium Clavadel (Davos : Suisse, Rudolf Gaberel architecte, 1930).

Phot. Grandvoinnet, Ph., 2004. (c) Philippe Grandvoinnet.

14 Cuvre emblématique de l'architecte Alvar Aalto, le sanatorium de Paimio ${ }^{13}$ (1928-1933) a progressivement été converti en hôpital à partir des années 1960 ; il dépend aujourd'hui des hôpitaux centraux de l'université de Turku. Le bâtiment d'hospitalisation et ses annexes ont été protégés en 1993 au titre de la loi finlandaise sur la conservation des édifices. L'ancien sanatorium n'a pas fait l'objet d'une campagne de restauration à proprement parler mais d'un programme exigeant d'entretien visant à préserver sur le long terme les caractéristiques architecturales des bâtiments (nature des matériaux, couleurs, mobilier), tout en assurant le bon fonctionnement des activités médicales qu'ils abritent ${ }^{14}$ (fig. 5). 
Figure 5

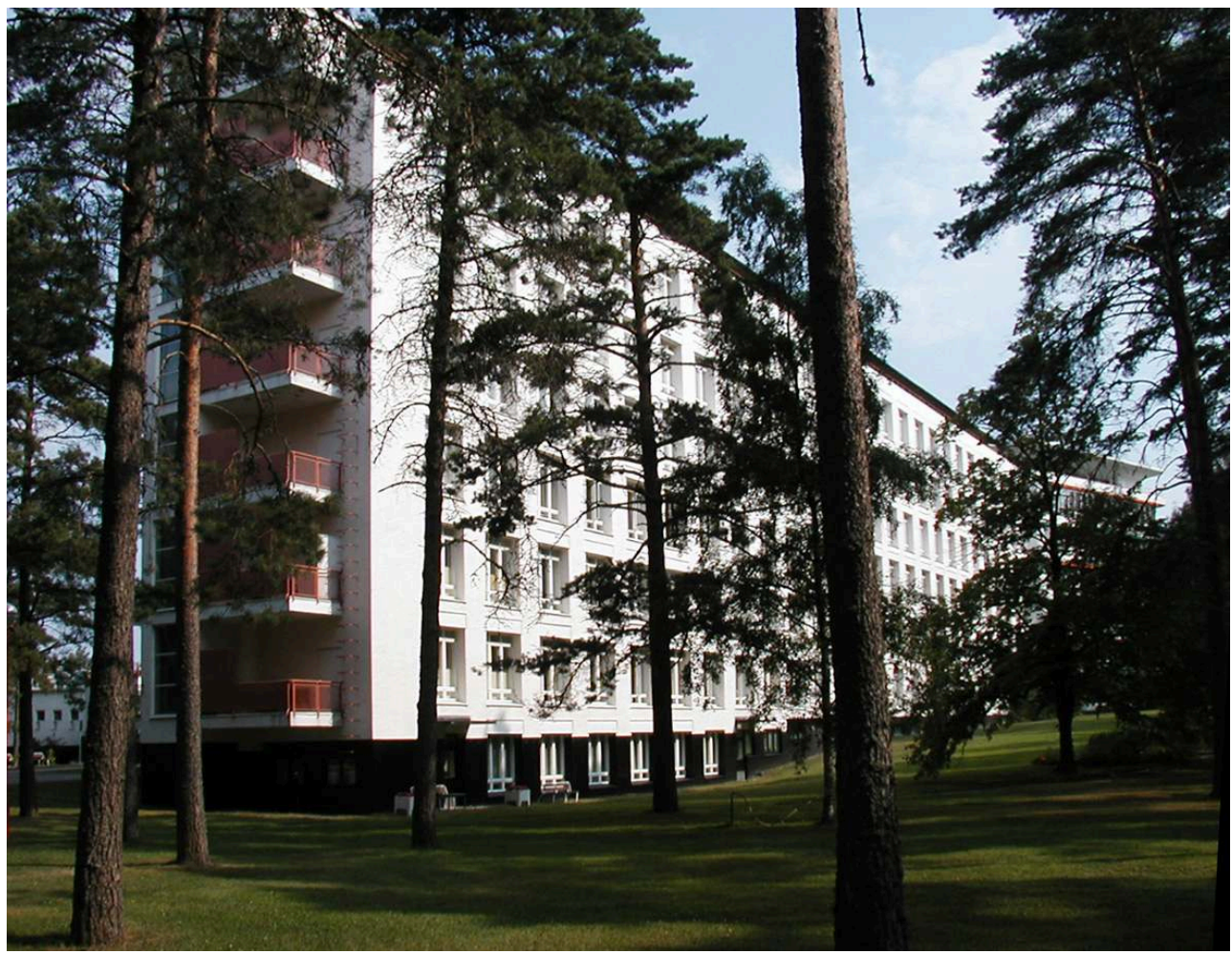

Sanatorium de Paimio (Finlande, Alvar Aalto architecte, 1933) : façade du bâtiment des chambres.

Phot. Grandvoinnet, Ph., 2003. ( ) Philippe Grandvoinnet.

Les critères de conservation des éléments présentant un intérêt architectural ou technique ont ainsi été intégrés au cahier des charges des interventions ; cela concerne notamment l'amélioration des conditions de confort et la mise aux normes des équipements. Ces interventions bénéficient de l'appui technique de la fondation Alvar Aalto et du Comité national des monuments historiques. Engagé par étapes, en fonction des besoins de l'établissement, de l'avancée des connaissances sur la production de l'architecte et des crédits disponibles, ce programme d'entretien s'apparente de fait à de la conservation préventive. Il garantit la cohérence globale du projet de conservation et la vision à long terme de ce qui ne serait autrement qu'une succession d'interventions ponctuelles (fig. 6). 
Figure 6

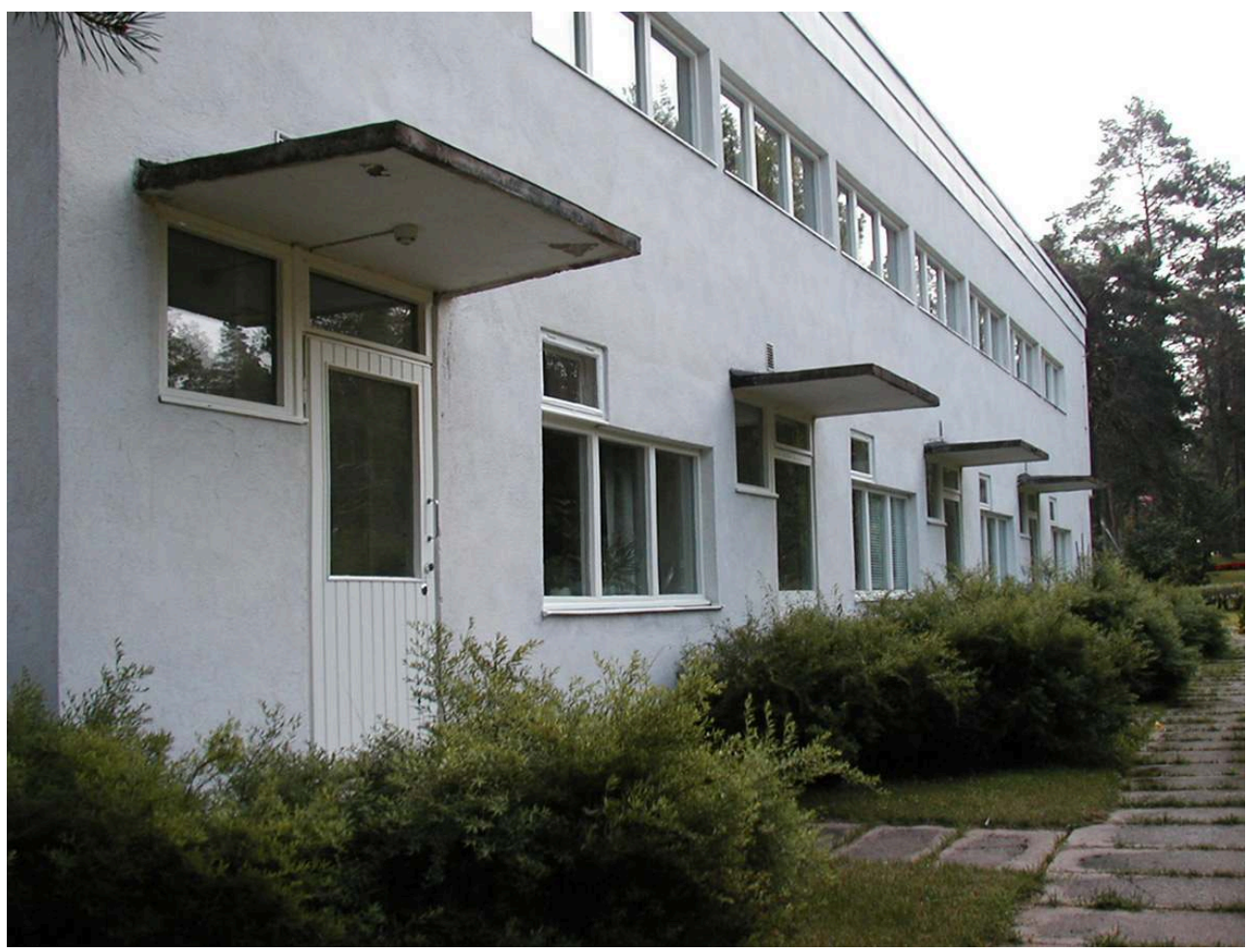

Sanatorium de Paimio, logements du personnel.

Phot. Grandvoinnet, Ph., 2003. ( ) Philippe Grandvoinnet.

Contemporain du sanatorium de Paimio, l'établissement Bella Lui - «belle lumière » (Montana, Suisse) a été construit à la fin des années 1920 par les architectes Rudolf Steiger et Flora Steiger-Crawford. Il met en œuvre une structure métallique innovante pour l'époque du fait de son mode d'assemblage par soudure (fig. 7). 


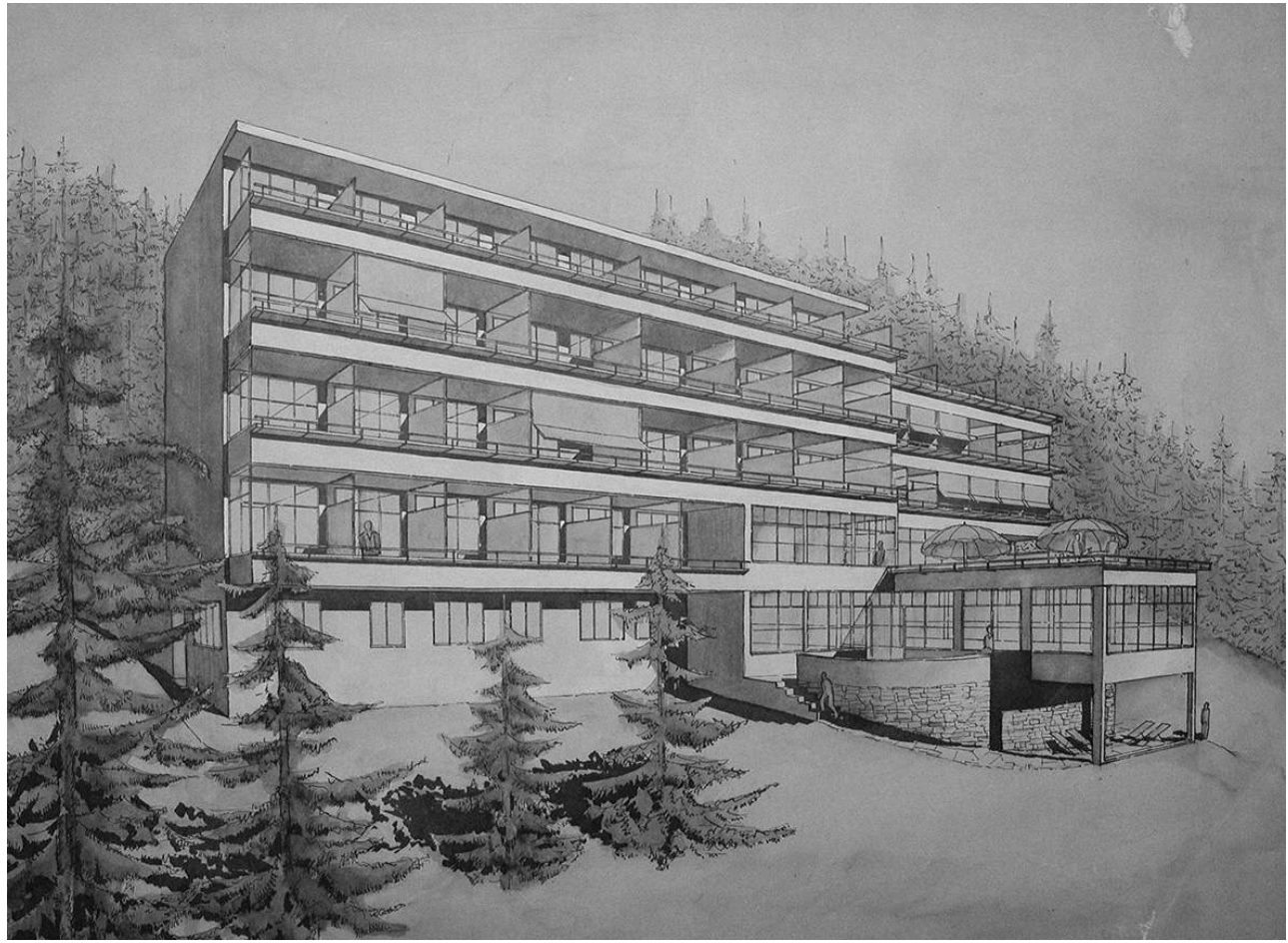

Sanatorium Bella Lui (Montana : Suisse, Rudolf Steiger et Flora Steiger-Crawford architectes, 1930) : perspective du projet (doc. GTA Archiv 100-051-F-In-29).

Du fait du développement de la station de ski de Montana, l'établissement a été transformé dès les années 1930 en " hôtel de sport » mais a conservé l'essentiel de ses aménagements d'origine, jusqu'à la réalisation d'une première campagne de travaux entre 1987 et 1992. Il s'agissait à cette époque de mettre l'établissement aux standards hôteliers, en créant notamment des salles de bains dans les chambres et en installant des cloisons phoniques. Le mobilier d'origine a disparu lors de ces travaux et les menuiseries en acier de la salle à manger ont été remplacées par des châssis en plastique. Programmé en 2001 dans la continuité de cette première intervention, le projet de « rénovation » des façades devait achever de remplacer les éléments d'origine par du matériel neuf. Cette deuxième tranche de travaux a pourtant donné l'occasion d'une réflexion globale sur la préservation des qualités architecturales du bâtiment, entre-temps repéré par le service des Monuments et des Sites du canton du Valais pour ses qualités architecturales remarquables. Le dialogue qui s'est alors engagé entre l'architecte Alfredo Orlando Piña, le propriétaire et l'administration cantonale a permis de définir le protocole d'un véritable projet de restauration de la façade sud du sanatorium, réalisée en 2003-2004 (fig. 8). Le bâtiment a d'abord été protégé afin que son propriétaire puisse bénéficier de subventions cantonales pour réaliser les travaux. L'architecte s'est ensuite appuyé sur l'expertise du service des Monuments et des Sites pour élaborer un projet de restauration fondé sur un diagnostic minutieux de l'existant. Ce diagnostic a permis de définir l'état initial de l'édifice (l'étude chromatique a par exemple montré que les façades étaient initialement couvertes d'une peinture minérale ocre jaune) et le type d'intervention nécessaire sur chaque élément de façade, l'objectif étant de conserver le plus de matière originale possible ${ }^{15}$. Cette intervention est le résultat d'un véritable projet d'architecture fondé sur une sélection minutieuse des éléments à conserver et de ceux pouvant être réparés ou remplacés. 
Même si cette restauration est limitée à la façade sud de l'édifice, la plus-value est globale: non seulement la qualité exceptionnelle du bien a été reconnue à cette occasion mais elle a permis au propriétaire-gestionnaire de repositionner son offre commerciale. Devenu «hôtel historique Bella Lui 1930 », l'établissement fait aujourd'hui partie du réseau Swiss historic hotels ${ }^{16}$ en tant que «témoin vivant de l'architecture moderne ».

\section{Figure 8}

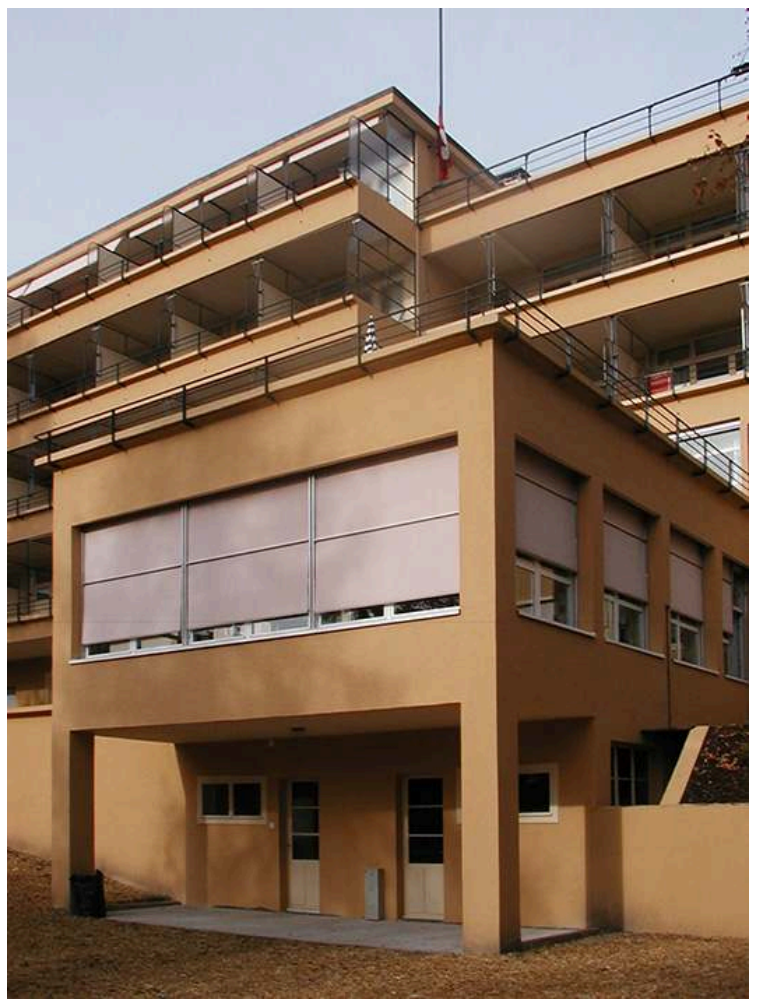

Sanatorium Bella Lui (Montana : Suisse, Rudolf Steiger et Flora Steiger-Crawford architectes, 1927-1930; restauration : Alfredo Orlando Piña architecte, 2005) : la façade sud restaurée.

Phot. Grandvoinnet, Ph., 2004. (c) Philippe Grandvoinnet.

Le projet de réhabilitation des sanatoriums de Beelitz (Schmieden \& Boetke architectes, 1898-1908), en Allemagne, constitue le rare exemple d'une intervention sur un ensemble sanatorial important. Construits au tournant $d u x^{e}$ siècle pour la caisse régionale d'assurance de Berlin, ces établissements forment un vaste complexe constitué de différentes sections (hommes et femmes, contagieux et non contagieux) et d'adjonctions successives comprenant au total plus de six cents lits répartis sur un site boisé de deux cents hectares (fig. 9, fig. 10). 
Figure 9

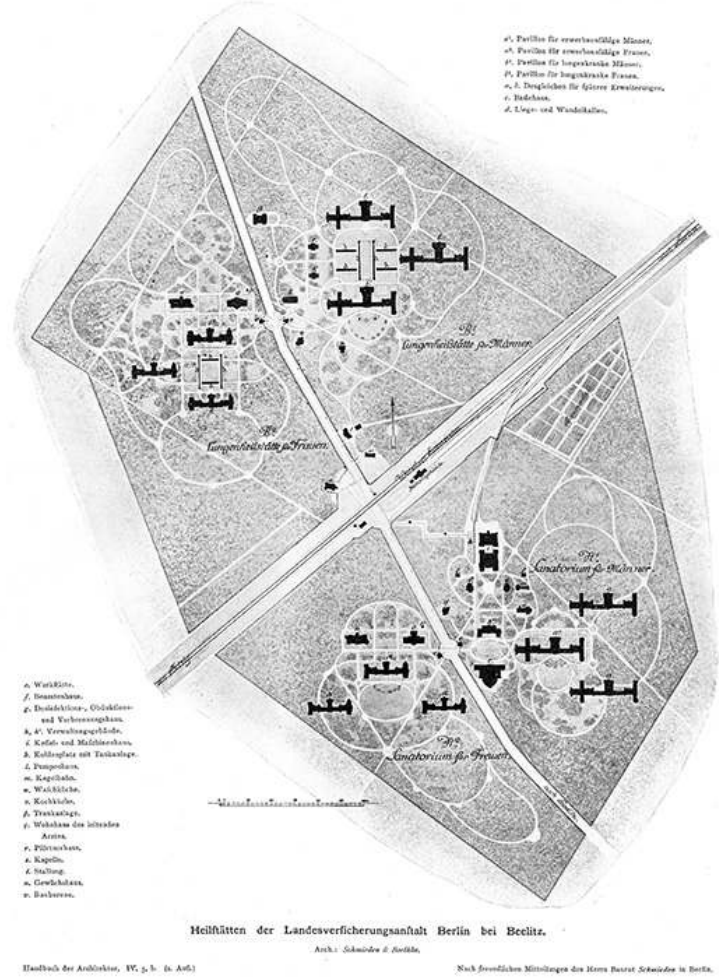

Sanatoriums de Beelitz (Allemagne, Schmieden et Boetke architectes, 1898-1908) : plan de masse. Dans Handbuch der Architektur, Stuttgart, 1903. 
Figure 10

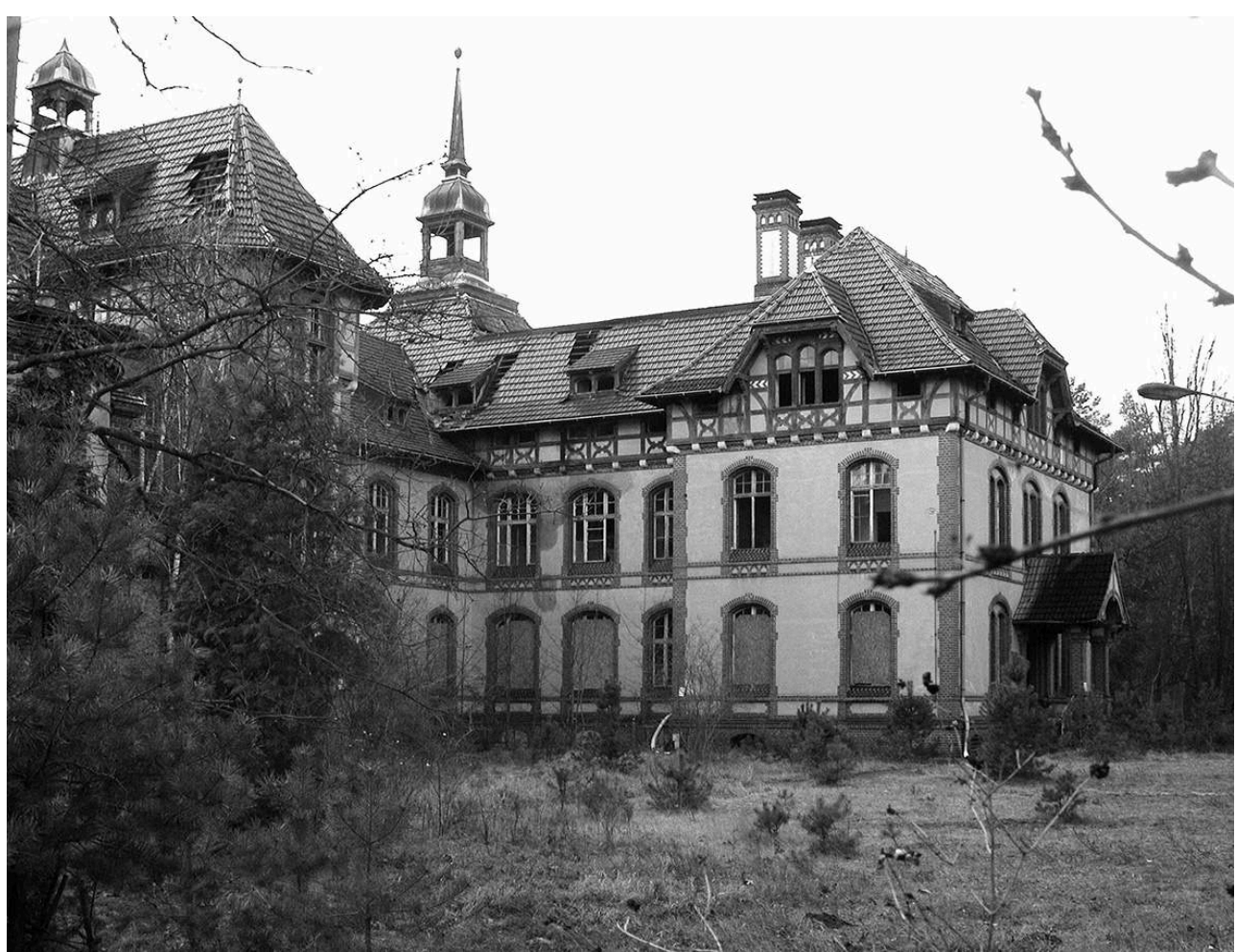

Sanatoriums de Beelitz, un bâtiment de cure à l'abandon.

Phot. Grandvoinnet, Ph., 2007. (c) Philippe Grandvoinnet.

Bombardé pendant la Seconde Guerre mondiale puis reconverti en hôpital pour l'armée soviétique, le site a été racheté en 1996 par un groupe immobilier qui mise sur la proximité de Berlin pour y développer un quartier mixte de logements, d'activités et de services. Les anciens sanatoriums se retrouvent au cœur d'un ambitieux projet de renouvellement urbain et de valorisation du patrimoine architectural. Les travaux engagés en 1998 annoncent la renaissance de Beelitz : le bâtiment de cure des hommes est transformé en clinique neurologique (fig. 11), les anciens logements des employés sont réhabilités et la chaufferie, véritable monument d'architecture et de technique, fait l'objet d'une restauration méticuleuse conduite par la conservation du patrimoine du district de Potsdam-Mittelmark avec l'aide des fonds structurels européens (fig. 12). 
Figure 11

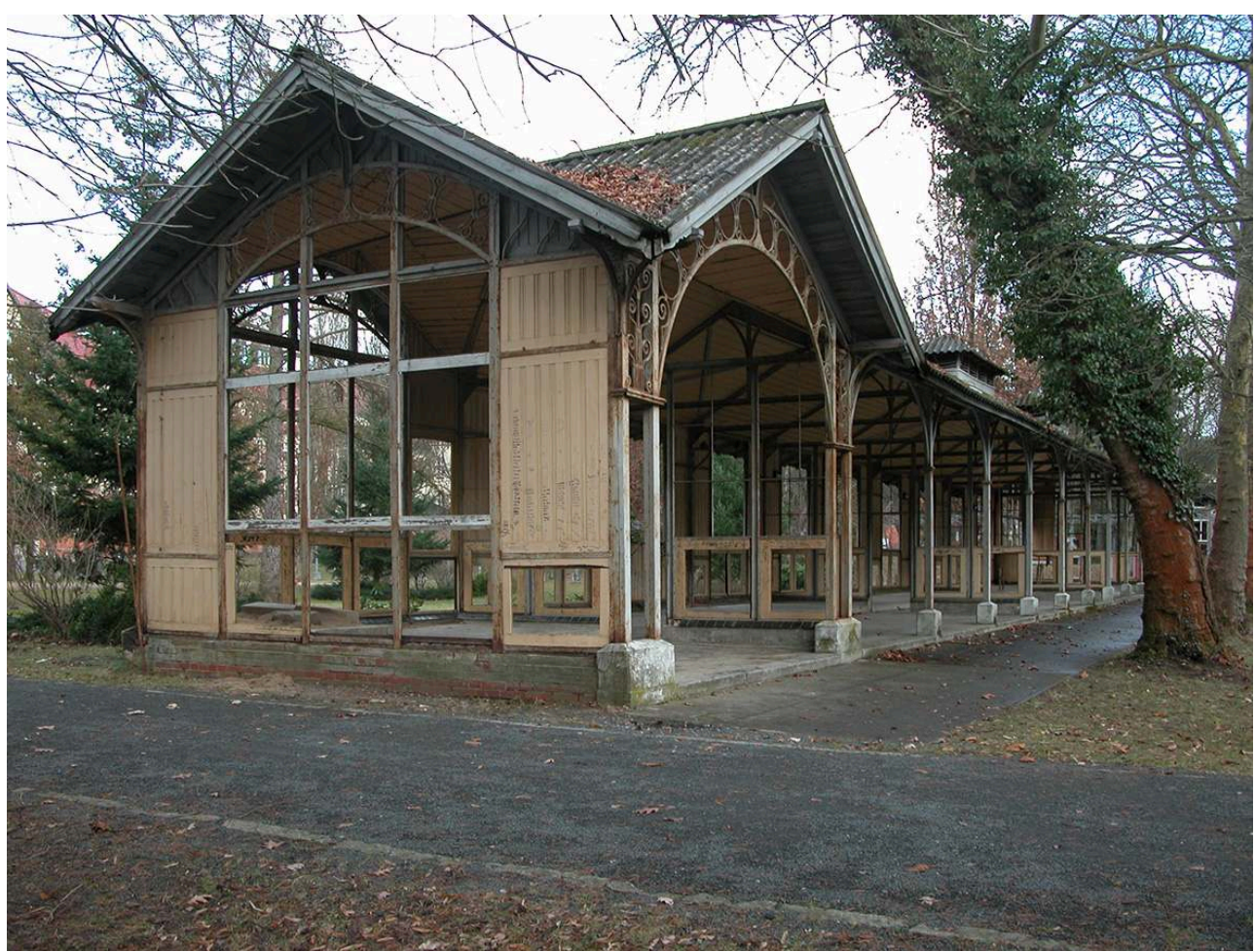

Sanatoriums de Beelitz, une galerie de cure.

Phot. Grandvoinnet, Ph., 2007. ( ) Philippe Grandvoinnet.

Figure 12

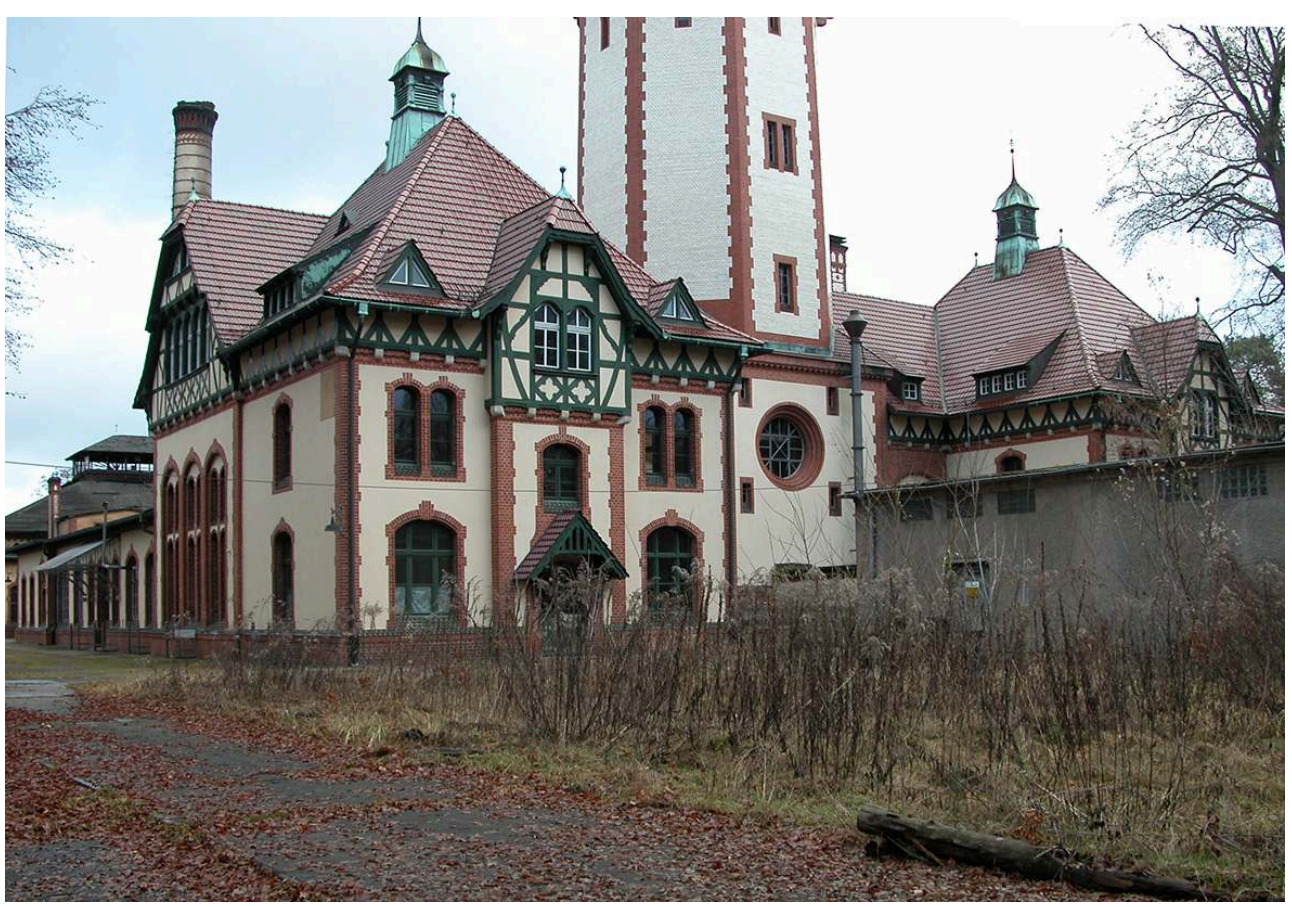

Sanatoriums de Beelitz, la chaufferie restaurée.

Phot. Grandvoinnet, Ph., 2007. ( ) Philippe Grandvoinnet. 
Le projet prévoit la restauration des jardins dans leur état initial ainsi que la construction de nouveaux quartiers d'habitat individuel. Mais la faillite de l'investisseur, au début des années 2000, marque un coup d'arrêt brutal pour ce projet qui peine aujourd'hui à redémarrer sur le plan immobilier en dépit d'initiatives multiples, tel l'aménagement d'un centre technique de sapeurs-pompiers dans les anciennes écuries du sanatorium. Paradoxalement, c'est un projet visant à exploiter le caractère spectaculaire des ruines de plusieurs bâtiments qui a donné un nouveau souffle au site. Porté par une société privée, le «sentier des cimes des sanatoriums de Beelitz » (Baumkronenpfad Beelitz-Heilstätten) a été inauguré en $2015^{17}$. Il comprend, sur la moitié nord du site, une passerelle métallique de cinq cents mètres de long placée à vingt mètres de haut au niveau de la cime des arbres. Le parcours proposé traverse l'ancien bâtiment de cure des femmes, totalement ruiné en 1945 et dont les hauts murs sont aujourd'hui couronnés par une étonnante forêt suspendue de pins (fig. 13), élément phare de la visite. Il se termine par l'ascension d'une tour panoramique en métal de quarante mètres de haut qui permet de contempler l'ensemble du site et, audelà, les forêts du Brandebourg, jusqu'à Berlin. Des visites guidées plus complètes permettent de dépasser cette vision fascinante du paysage offert par les ruines, en donnant notamment accès à plusieurs bâtiments désaffectés, tel l'ancien pavillon de chirurgie construit en 1930. Ce projet renouvelle le regard du visiteur sur un ensemble sanatorial conçu pour former un ensemble médical cohérent. Il considère également le site (forêt et bâtiments) pour ses qualités propres de paysage recomposé par le temps, prenant le contrepied de la vision idéalisée du parc sanatorial d'origine ${ }^{18}$.

Figure 13

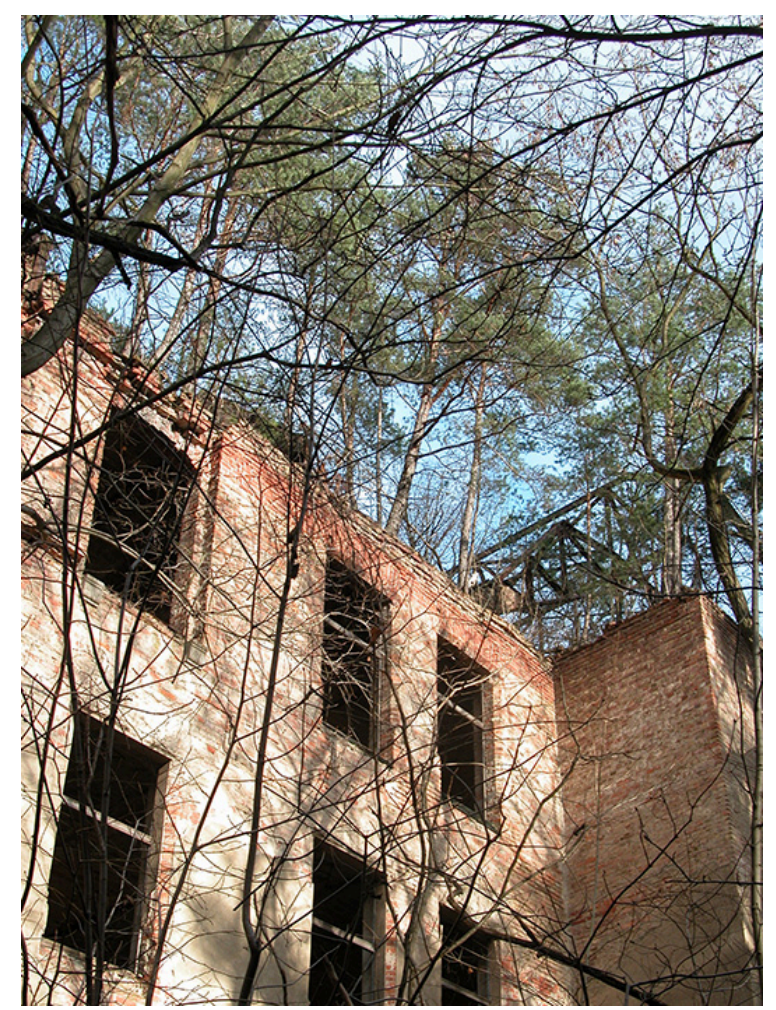

Sanatoriums de Beelitz, le sanatorium des femmes en ruine. Phot. Grandvoinnet, Ph., 2007. (c) Philippe Grandvoinnet. 


\section{Réhabilitations pragmatiques}

21 En France, où la production sanatoriale fut sans doute la plus importante et la plus variée d'Europe durant l'entre-deux-guerres, seuls trois sanatoriums pour tuberculeux pulmonaires ont été protégés au titre des monuments historiques. Bien que tous construits au début des années 1930, ils sont représentatifs de la diversité de ce type de constructions de l'époque par leur situation et les catégories de malades auxquelles ils s'adressaient: Aincourt (1929-1933) était un sanatorium populaire à la campagne, Sabourin (Puy-de-Dôme, 1929-1934) un hôpital-sanatorium péri-urbain, et Martel de Janville (Haute-Savoie, 1932-1935) un sanatorium militaire d'altitude.

Construit sur le plateau d'Assy par les architectes Pol Abraham et Henry Jacques Le Même, le sanatorium Martel de Janville a été désaffecté en 2006 par son propriétaire historique, l'Association des villages sanatoriums de haute altitude, et protégé (façades et toitures, ainsi que la chapelle dans son intégralité) peu de temps après. Les contraintes architecturales posées en matière de réhabilitation par cette protection font l'objet de négociations entre l'État et le promoteur qui a acquis entre-temps le bâtiment. Le programme immobilier prévoit l'aménagement d'une résidence de tourisme de cent vingt appartements dans les cent soixante chambres et les espaces collectifs, techniques et médicaux existants. Idéalement situé face au mont Blanc, l'attractivité de l'ancien sanatorium tient aussi à sa qualité de monument historique qui lui donne le statut convoité de produit fiscal ${ }^{19}$. Le projet de réhabilitation, confié à l'architecte Marc Rolinet, est complexe à mettre en œuvre en raison du fragile équilibre entre le modèle économique de l'opération et les exigences liées à la protection. Si la restauration de la façade sud - la plus emblématique - dans son état d'origine semble aller de soi, il n'en va pas de même des autres façades. Le promoteur obtient ainsi d'augmenter l'habitabilité des ailes de cure par l'adjonction de circulations horizontales en façade nord, ce qui permet d'affecter la totalité les chambres et des couloirs existants aux nouveaux logements. L'architecte doit également faire preuve d'ingéniosité pour affecter la quasi-totalité des surfaces existantes à du logement : la généreuse façade sud du bâtiment cache en effet des corps de bâtiments épais et mal orientés. La réutilisation des majestueux espaces collectifs du sanatorium pose plus de difficultés encore dans un programme exclusivement dédié au logement: il faut soit leur trouver un usage qui assure la préservation de leur intégrité architecturale et permette à chaque occupant d'en jouir (c'est le cas de la chapelle, protégée au titre des monuments historiques et transformée en salle d'exposition), soit se résoudre à voir disparaître leur usage collectif. La force architectonique de la salle à manger (dont les arcs en béton armé portent une partie de la façade sud de l'édifice) a ainsi été perdue dans le découpage opéré pour y aménager des logements en duplex. Ce projet, en dépit de ses qualités et de l'accompagnement réalisé par le service des Monuments historiques, pose la question de l'adéquation du programme avec l'existant et de l'incidence des compromis négociés entre l'aménageur et l'administration. L'essentiel, toutefois, a été sauvegardé : l'insertion paysagère du bâtiment, la façade sud, surtout, qui a retrouvé sa teinte "terre cuite claire » d'origine et a conservé ses menuiseries anciennes (châssis à double guillotine actionnée par contre-poids), dont les performances (étanchéité à l'air, isolation thermique, manœuvrabilité) ont été simplement améliorées (fig. 14). La réhabilitation du bâtiment, dont la variété des 
espaces disponibles est l'une des caractéristiques, aurait toutefois sans doute été facilitée par un programme intégrant une plus grande mixité fonctionnelle (permettant de répartir les besoins en fonction des caractéristiques de l'existant) et un meilleur équilibre entre les espaces collectifs et les espaces privés.

Figure 14

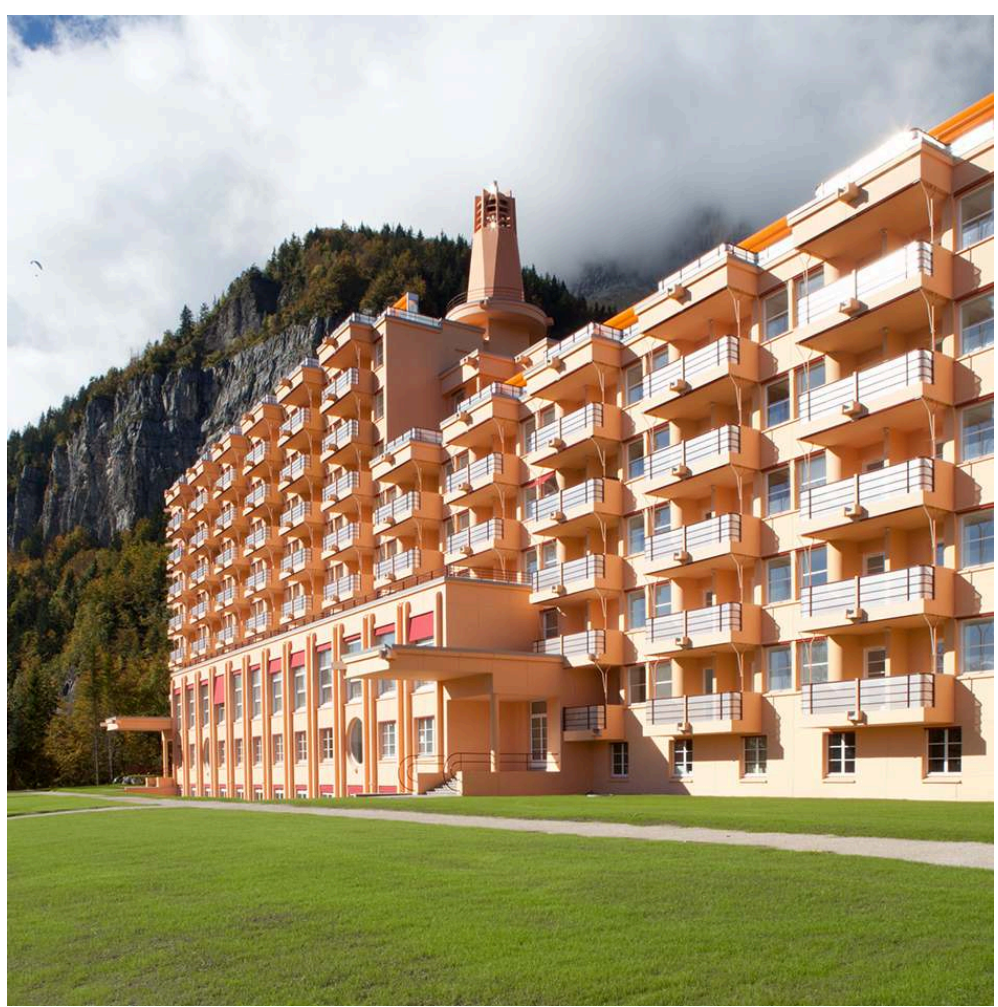

Sanatorium Martel de Janville (Plateau d'Assy : Haute-Savoie, Pol Abraham et Henry Jacques Le Même architectes, 1937) : façade sud après restauration.

Phot. CAUE 74. @ Romain Blanchi-caue74.

Le sanatorium populaire d'Aincourt (Paul Decaux \& Édouard Crevel architectes) est une autre illustration des difficultés posées par la typologie sanatoriale en matière de réhabilitation ${ }^{20}$ (fig. 15, fig. 16). Il constitue l'un des rares exemples en Europe, avec l'établissement allemand de Waiblingen (Richard Döcker architecte, 1929) et le sanatorium Guébriant (plateau d'Assy, Abraham \& Le Même architectes, 1932), de sanatorium construit selon le système à gradins pour des raisons d'exposition des chambres et d'accessibilité des terrasses de cure $^{21}$. Deux des trois pavillons du sanatorium d'Aincourt ont été protégés au titre des monuments historiques en 1999 et ont fait l'objet d'études préalables à leur restauration. Comme à Zonnestraal, les investisseurs misaient en quelque sorte sur la qualité paysagère du site et la proximité de Paris (à soixante kilomètres) pour les convertir en immeubles de logement. Le projet à l'étude est ambitieux puisqu'il prévoit la restauration des pavillons en l'état d'origine, sans extension ni modification de la volumétrie; seules les circulations internes seraient modifiées pour pallier la faible épaisseur des corps de bâtiment. L'organisation des logements en duplex permettra de gagner sur les couloirs tout en préservant, sur un ou deux niveaux, la distribution existante. Il ne s'agit toutefois là que d'intentions, fondées sur une analyse précise de l'existant et de son potentiel de remploi, mais 
susceptibles d'être remises en question par les contraintes programmatiques, économiques ou techniques qui pourront survenir.

Figure 15

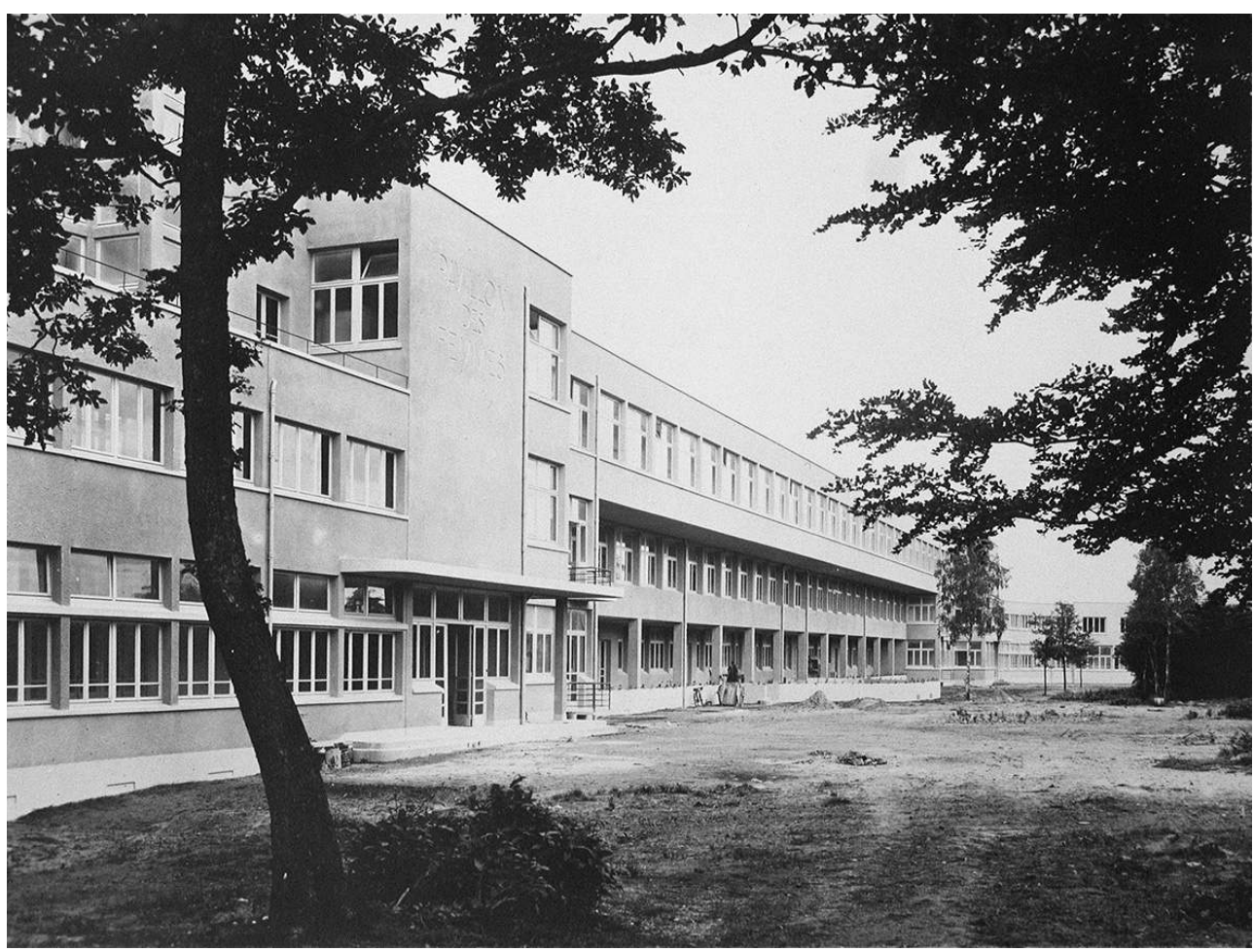

Sanatorium d'Aincourt (Val-d'Oise : France, Paul Decaux et Édouard Crevel architectes, 1930-1933) : façade nord du pavillon Vian. Archives départementales du Val-d'Oise.

(c) Archives départementales du Val-d'Oise. 


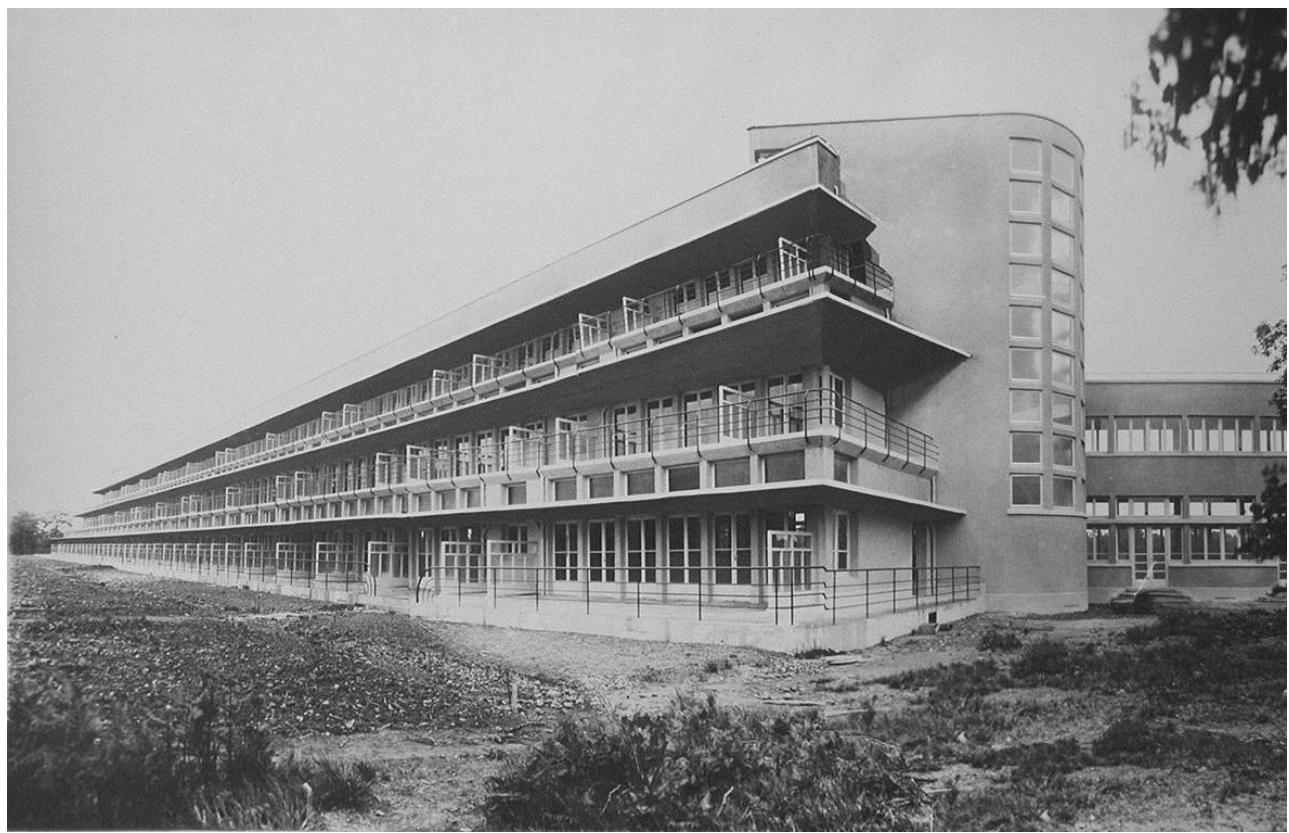

Sanatorium d'Aincourt : façade sud du pavillon Amiard. Archives départementales du Val-d'Oise.

(c) Archives départementales du Val-d'Oise.

\section{L'hôpital-sanatorium Sabourin, un cas d'école}

L'établissement fut construit entre 1929 et 1934 pour les hospices de Clermont-Ferrand par l'architecte Albéric Aubert ${ }^{22}$. La mise en œuvre d'une ossature en béton armé permet la superposition de niveaux très différents dans leur organisation (dortoirs et chambres, avec ou sans galerie de cure, à façade plane ou à redents), conçus pour répondre à la diversité des situations des malades et de leurs pathologies (fig. 17). Cette réalisation unique est une sorte de pot-pourri, une sélection de ce qui se fait alors de mieux en matière de conception sanatoriale : on y retrouve les dortoirs cloisonnés en boxes (c'est une spécialité française depuis le début du $\mathrm{xx}^{\mathrm{e}}$ siècle), mais aussi les expériences plus audacieuses des architectes Pol Abraham et Henry Jacques Le Même pour le sanatorium de Plaine-Joux (non réalisé) et de Guébriant au plateau d'Assy. Endommagé lors du bombardement des usines Michelin en 1944, le sanatorium réouvre en 1947. À partir des années 1960, l'activité médicale de l'établissement évolue vers la pneumo-allergologie : l'« hôpital-sanatorium » devient " hôpital » et connaît une baisse inexorable de son activité, jusqu'à sa fermeture définitive en 1997. Sa démolition est alors envisagée mais un collectif d'architectes et d'historiens obtient du ministère de la Culture en 1999 une instance de classement. Cette procédure permet d'interrompre tout projet pendant un an et d'étudier l'opportunité d'une protection. En 2000, le sanatorium est inscrit au titre des monuments historiques avec son jardin et ses bâtiments annexes ${ }^{23}$ : l'intérêt historique et architectural de l'ensemble ne fait aucun doute, et c'est avec Martel de Janville et Aincourt l'un des rares sanatoriums encore intacts en France. L'ensemble est acheté en 2002 par l'État, qui envisage d'y transférer l'école d'architecture de Clermont-Ferrand et ses 600 étudiants. Désaffecté, le bâtiment est la victime d'un vandalisme sauvage et il n'en reste que la structure en béton armé et une partie des menuiseries lorsque le concours d'architecture pour sa réhabilitation est 
lancé en 2007. Le programme pour la transformation de l'ancien sanatorium en école d'architecture prévoit l'aménagement de $7000 \mathrm{~m}^{2}$ de plancher pour un budget prévisionnel d'environ 30 millions d'euros.

Figure 17

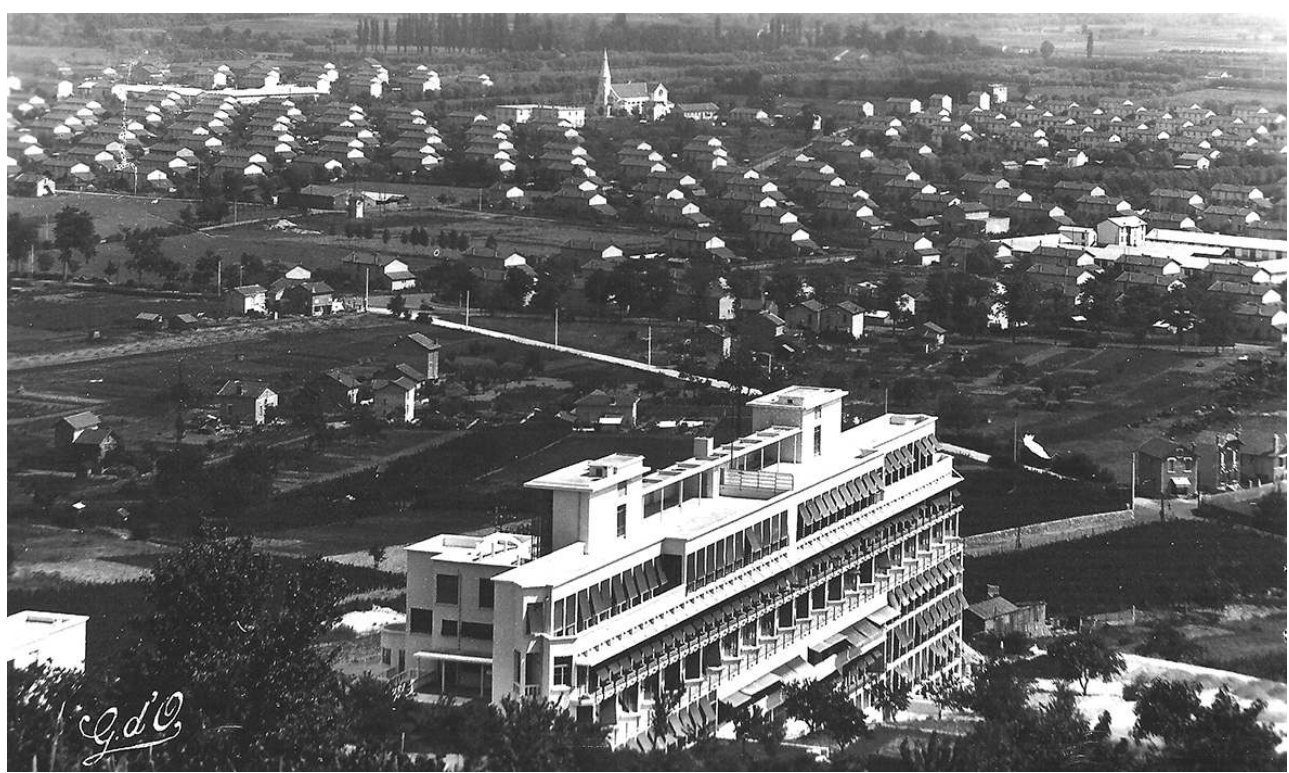

Hôpital-sanatorium Sabourin (Clermont-Ferrand : Puy-de-Dôme, Albéric Aubert architecte, 1934) : vue générale du sanatorium et des quartiers ouvriers. Carte postale, années 1930, coll. part.

$\mathrm{Du}$ fait de la protection dont il fait l'objet, une «notice d'orientations et de prescriptions d'ordre patrimonial et historique » est annexée au cahier des charges du concours afin d'aider les candidats à élaborer une proposition conforme aux objectifs de mise en valeur de cette réalisation emblématique de l'architecture du $\mathrm{xx}^{\mathrm{e}}$ siècle en Auvergne. La notice insiste sur la relation du bâtiment à son environnement et précise notamment que « toute addition d'espaces construits le sera dans le strict respect de ce lien indissociable entre bâti et jardin qui structure fortement le domaine $»^{24}$, c'est-àdire dans un rapport d'effacement vis-à-vis de l'existant. Elle ne donne toutefois aucune indication sur les caractéristiques d'usage, spatiales ou techniques du bâtiment, ni sur l'opportunité d'en maintenir la lisibilité dans le projet. Ces éléments sont laissés à l'appréciation des candidats, invités en toute logique à "se conformer à l'écriture moderne et fonctionnaliste » qui caractérise l'édifice.

Les architectes lauréats du concours, Pierre du Besset et Dominique Lion, ont fait le choix de restituer l'aspect d'origine de la façade sud du bâtiment - emblématique de sa vocation thérapeutique initiale - et d'adapter les autres parties aux besoins du programme. La transformation d'un établissement de santé en établissement d'enseignement est un défi, car tout oppose ces deux programmes en termes d'espace (fig. 18, 19, 20, 21). 
Figure 18

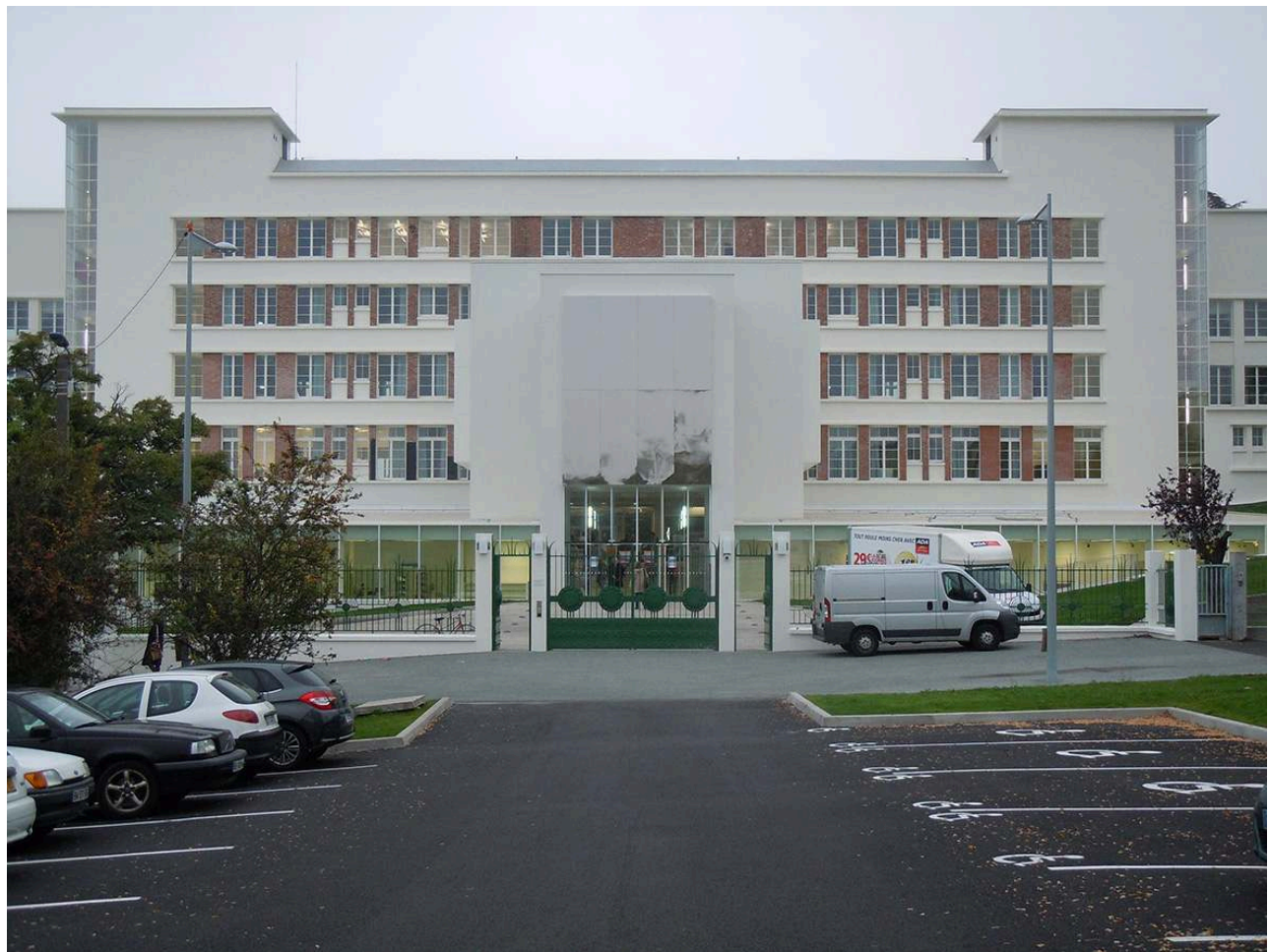

École nationale supérieure d'architecture de Clermont-Ferrand, ancien hôpital-sanatorium Sabourin (projet réhabilitation : Pierre du Besset et Yves Lion architectes, 2007-2015) : la façade nord de l'ENSA. Phot. Grandvoinnet, Ph., 2015. (c) Philippe Grandvoinnet. 
Figure 19

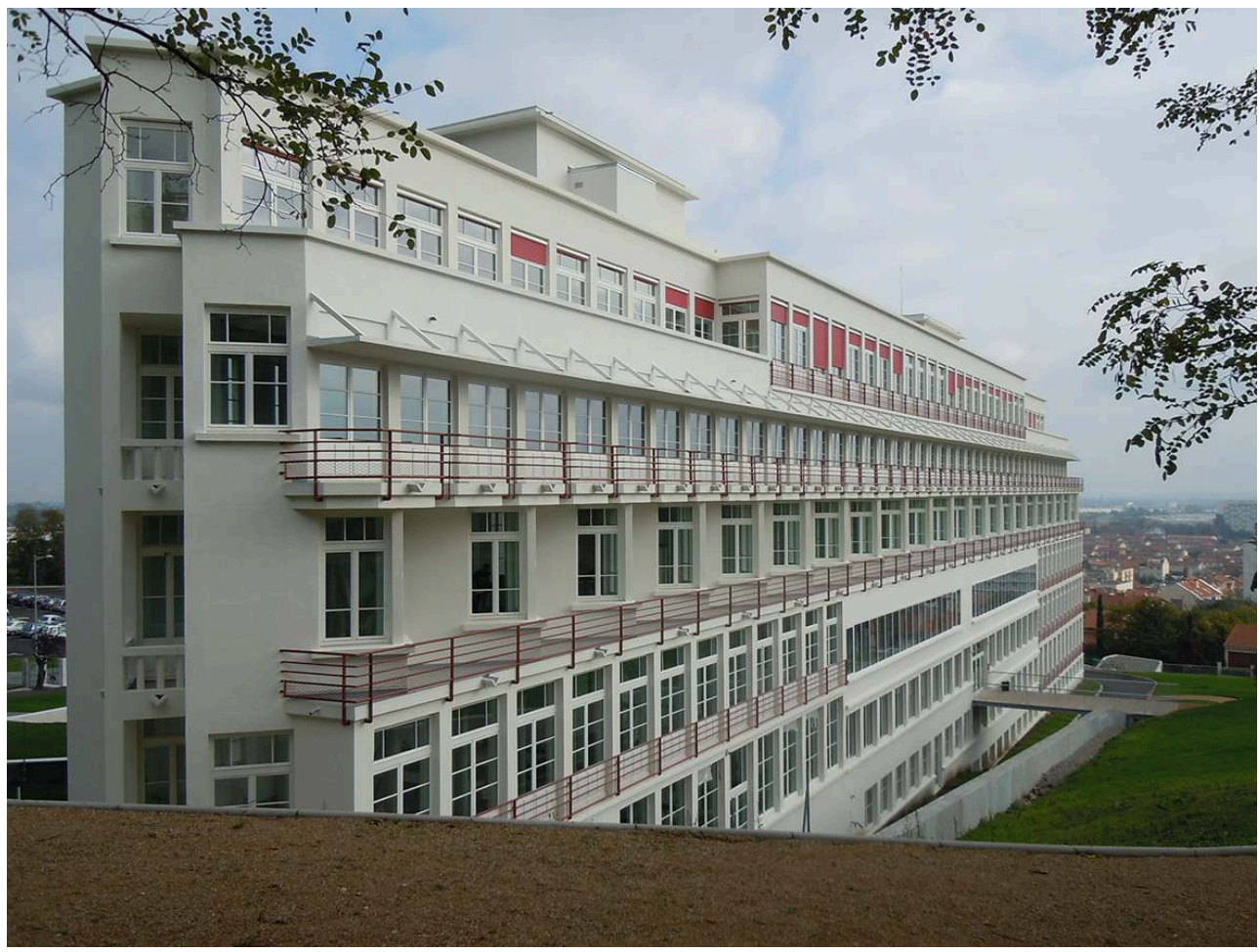

École nationale supérieure d'architecture de Clermont-Ferrand : façade sud restaurée de l'ancien sanatorium.

Phot. Grandvoinnet, Ph., 2015. (c) Philippe Grandvoinnet. 
Figure 20

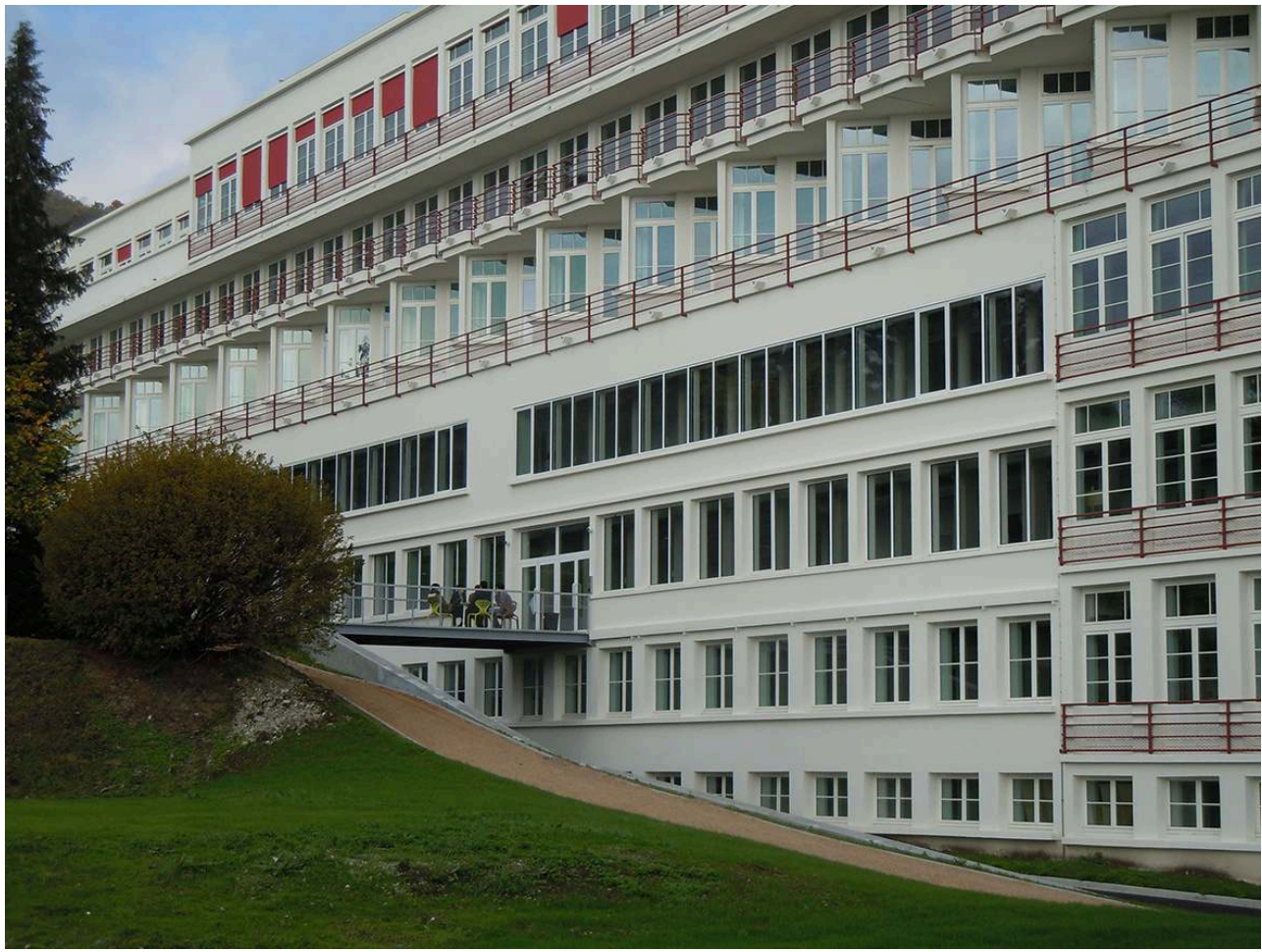

École nationale supérieure d'architecture de Clermont-Ferrand : façade sud restaurée de l'ancien sanatorium.

Phot. Grandvoinnet, Ph., 2015. ( ) Philippe Grandvoinnet. 
Figure 21

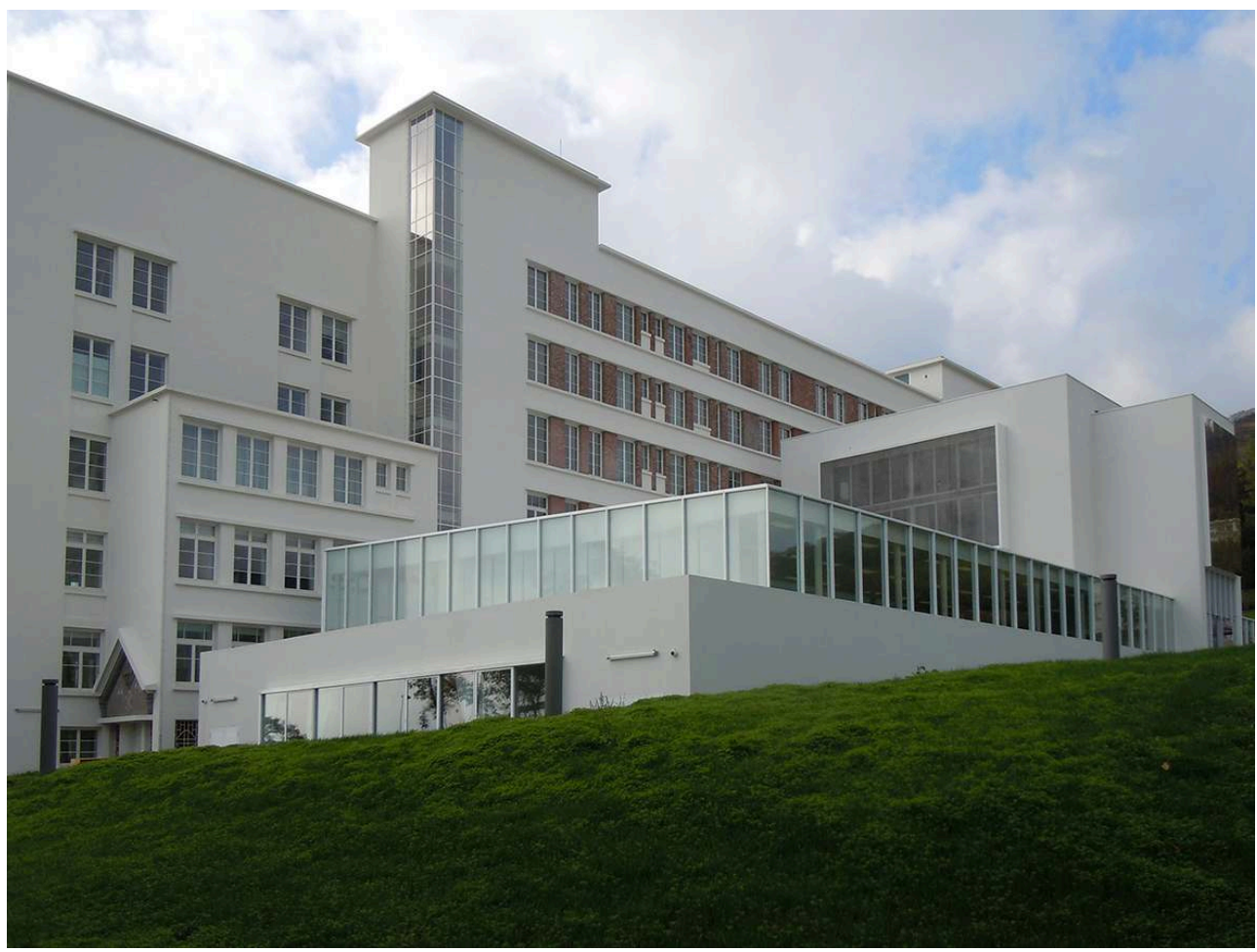

École nationale supérieure d'architecture de Clermont-Ferrand : extensions en façade nord.

Phot. Grandvoinnet, Ph., 2015. (c) Philippe Grandvoinnet.

Ils convergent toutefois sur un point : la nécessité de démontrer le caractère opératoire de leurs dispositions architecturales, à vocation thérapeutique pour l'un, pédagogique pour l'autre. Les contradictions typologiques entre les deux programmes se sont trouvées aggravées par des contraintes techniques complexes, liées notamment à l'accessibilité du bâtiment et aux contraintes réglementaires liées au changement d'affectation. La problématique soulevée par la résistance parasismique du bâtiment constitué d'un corps long, peu épais et en effet faiblement contreventé - a ainsi conduit les architectes à choisir une solution technique radicale : reconstruire intégralement la structure interne de l'édifice sous la forme d'une ossature parasismique tridimensionnelle en béton et en métal. Pour que le monument respecte les normes parasismiques en vigueur, il a fallu le vider de sa structure d'origine et donc de l'essentiel de ses aménagements intérieurs (fig. 22). 
Figure 22

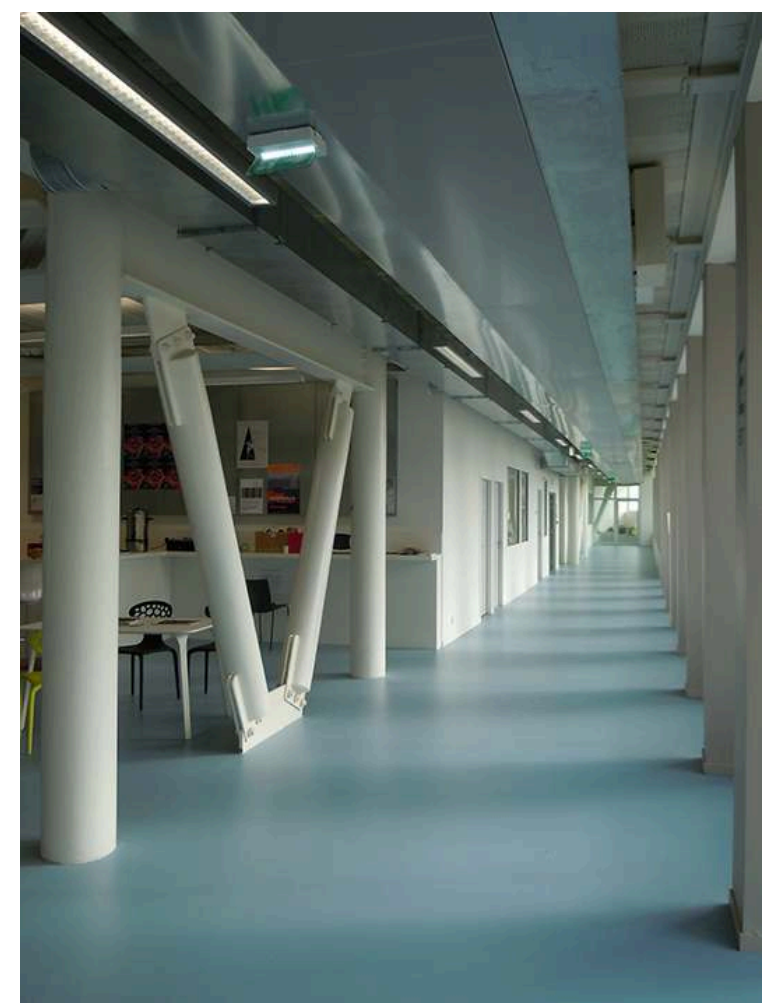

École nationale supérieure d'architecture de Clermont-Ferrand : nouvelle structure interne.

Phot. Grandvoinnet, Ph., 2015. (c) Philippe Grandvoinnet.

Du fait de la configuration de l'aile de cure (exposition plein sud, grande longueur, faible épaisseur) la distribution générale du bâtiment a également été modifiée. Elle se caractérisait initialement par des couloirs placés au nord qui distribuaient les chambres et les dortoirs exposés au sud. Cette conception climatique, acceptable en régime de "surventilation » des espaces propre à un sanatorium ne permettait sans doute pas de transformer tels quels les anciens dortoirs de malades en salles d'enseignement: exposés au sud, ces espaces connaissent quotidiennement de fortes variations d'ensoleillement, à quoi s'ajoutent des écarts importants de température d'une saison à l'autre. La maîtrise du confort climatique des espaces d'enseignement se heurte également à l'exigence de conservation des menuiseries épargnées par les actes de vandalisme sur la façade sud (un tiers du total environ). Les architectes ont donc fait le choix d'inverser la distribution en reportant au sud le couloir qui dessert chaque niveau. Ces longs dégagements, espaces intermédiaires entre la façade sud restaurée et les salles de cours, forment une zone thermique qui régule les excès du climat extérieur. Les salles placées à l'arrière bénéficient au sud de la lumière diffuse des cloisons translucides et au nord d'un éclairage direct. Cette disposition permet à la fois de conserver un rôle actif - bien que détourné - à la façade méridionale, et d'assurer un bon niveau de confort (thermique et lumineux) aux espaces situés à l'arrière.

29 Ce projet illustre les difficultés posées par la nécessaire sélection de ce qui peut être préservé et de ce qui doit évoluer pour répondre à un nouvel usage : à Sabourin, la préservation des qualités architecturales de la façade sud, spécifiquement sanatoriale et à laquelle on reconnaît une valeur patrimoniale, a en quelque sorte légitimé les interventions plus lourdes réalisées sur les autres parties du bâtiment (destruction de 
l'aile nord, adjonction de nouveaux volumes de part et d'autre). Ce compromis serait sans doute passé inaperçu si la façade nord de l'établissement avait été une façade arrière, ce qu'elle n'est jamais dans un sanatorium.

Ces projets, dans leur diversité, ont permis de valoriser un patrimoine présentant de grandes qualités architecturales. Dans ces exemples, la protection au titre de monuments historiques a constitué un puissant levier de valorisation du patrimoine du $\mathrm{xx}^{\mathrm{e}}$ siècle, en raison du cadre réglementaire qu'elle impose et du suivi technique assuré par les services du patrimoine. Ces réhabilitations ne doivent toutefois pas masquer les difficultés dans lesquelles se trouve aujourd'hui le patrimoine sanatorial: les innombrables établissements récemment défigurés, désaffectés voire démolis, permettent de mesurer l'immense déficit en matière de valorisation. La conservation intégrale est un objectif sans doute inatteignable dans la plupart des cas; la hiérarchisation des enjeux patrimoniaux doit alors faire partie du cahier des charges donné au maître d'œuvre pour servir de guide à l'élaboration du projet: valeur architecturale, sociale, technique, décorative ou historique des éléments en place, risques de détérioration ou de perte de substance, capacité à s'intégrer à une nouvelle économie de projet, etc.

\section{Cités sanatoriales et patrimoines climatiques : leviers de développement local}

31 La question de la réhabilitation des anciens sanatoriums se pose en des termes différents selon qu'ils sont isolés ou groupés au sein de stations climatiques de cure. Dans ce dernier cas, seul un inventaire global et méticuleux permet d'évaluer le potentiel de la station dans les différents secteurs constitutifs de son économie: tourisme, santé, services, etc. En Suisse par exemple, la crise de l'activité sanatoriale s'est fait sentir plus tôt qu'ailleurs en Europe: en 1945, l'étude conduite par Armin Meili sur l'« assainissement technique des hôtels et des stations touristiques » reposait déjà sur un inventaire précis de l'hôtellerie et des établissements de cure existants. Elle a conduit à l'élaboration d'un plan d'aménagement global des anciennes stations climatiques ${ }^{25}$ (fig. 23). Pour chacune de ces stations, « il a fallu trouver des solutions de détail aux questions architecturales en tenant compte de la diversité des régions, de leur histoire, de leur culture, de leur économie $»^{26}$. Réalisé pour le compte de l'office fédéral des transports par les architectes les plus compétents dans ce domaine (tels Rudolf Gaberel à Davos ou Georges Épitaux à Leysin), ce rapport proposait une approche urbaine fondée sur la définition de zones distinctes (habitations, sanatoriums, verdure), l'analyse du trafic (dessertes existantes, voies nouvelles) et des constructions (nature des équipements à créer, maintien d'une unité architecturale, etc.). L'étude de chaque station était suivie d'un diagnostic urbain et de propositions censées aider les stations à repositionner leur offre et à préparer leur développement futur. Dans le cas de Leysin, les architectes proposèrent par exemple de séparer plus nettement le village de la station médicale, d'adopter un règlement pour la protection des espaces naturels, d'améliorer le réseau routier ou encore de construire de nouveaux équipements communautaires et sportifs ${ }^{27}$. Les stations climatiques suisses firent précocement le pari d'un recul massif et durable de la tuberculose, qui ferait perdre à court terme leur clientèle aux sanatoriums de cure. Cette position fut suivie en France par certaines stations : à Briançon, le périmètre d'une zone climatique est défini 
dès 1946 afin de mieux accompagner le développement du tourisme, de la station de Serre-Chevalier notamment. La plupart des autres stations de cure, notamment alpines, eurent toutefois plus de difficultés à sortir de la mono-activité médicale et climatique qui les caractérisait.

Figure 23

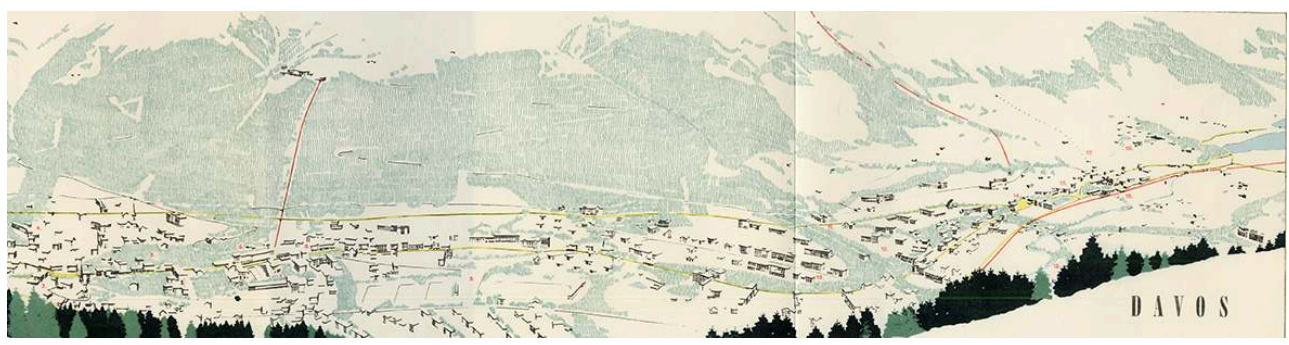

Davos, projet d'aménagement de la station, 1945. Dans Armin Meili, Assainissement technique d'hôtels et de stations touristiques, 1945.

En raison de la place qu'elles ont occupée dans la lutte contre la tuberculose, de la densité d'établissements qu'elles représentent et des formes urbaines spécifiques qui s'y sont développées, les anciennes stations climatiques de cure concentrent les enjeux les plus importants en matière de valorisation du potentiel économique et paysager de ce patrimoine climatique. Ces ensembles forment des paysages urbains homogènes, uniques en montagne et parfaitement intégrés à leur environnement. Leur avenir passe sans doute par une intégration plus forte aux réseaux économiques et touristiques régionaux, par l'intermédiaire notamment de documents d'urbanisme adaptés. À Briançon encore, le label «Ville d'art et d'histoire» est mobilisé depuis quelques années pour définir une stratégie de valorisation du patrimoine climatique dans un objectif d'élargissement de l'offre culturelle, trop longtemps centrée sur le seul patrimoine fortifié hérité de Vauban (fig. 24).

Figure 24

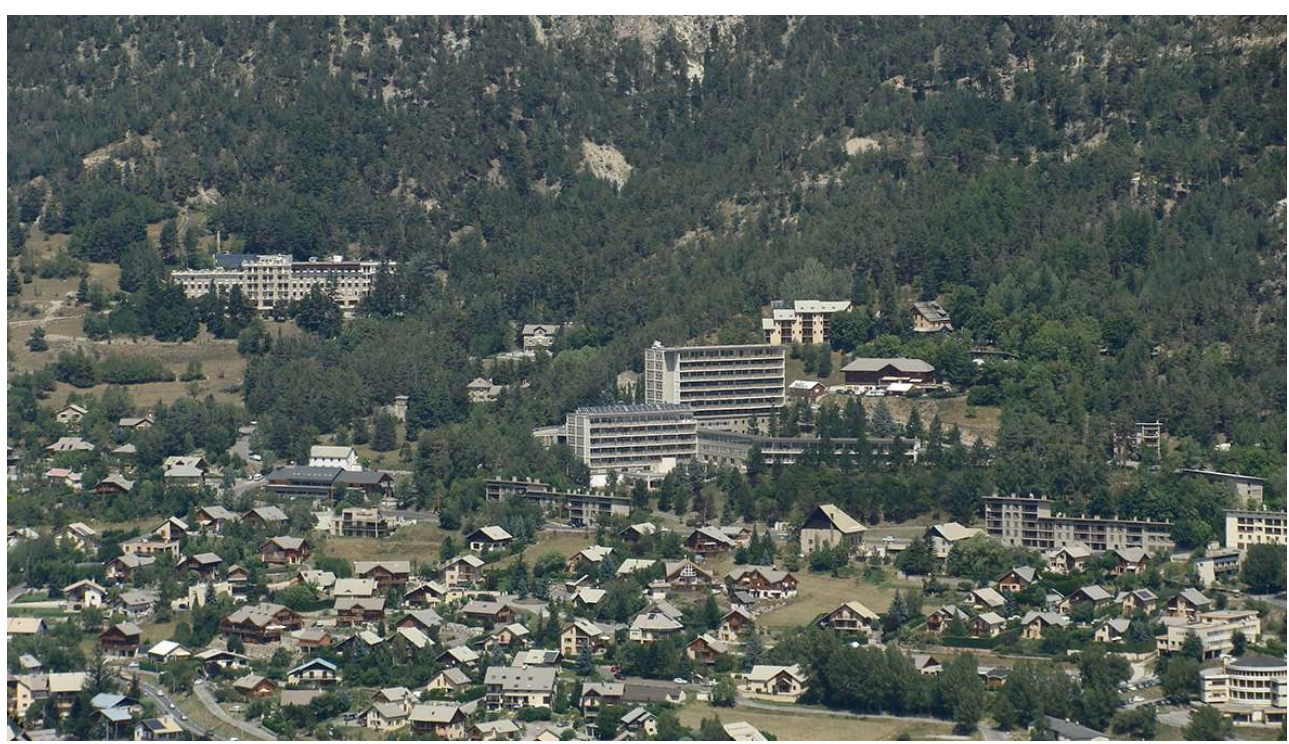

Briançon (Hautes-Alpes) : vue partielle de l'ancienne zone climatique de la station.

Phot. Grandvoinnet, Ph., 2015. (C) Philippe Grandvoinnet. 
En misant sur la reconnaissance apportée par le label « Patrimoine du $\mathrm{xx}^{\mathrm{e}}$ siècle », la municipalité, avec l'aide des services de l'État (DRAC-UDAP) a posé les jalons d'une politique culturelle et d'aménagement de l'espace qui s'appuie largement sur la mise en valeur du patrimoine, qu'il soit ancien ou récent (fig. 25). Au Plateau d'Assy (HauteSavoie) et à Hauteville (Ain), on s'interroge aujourd'hui de la même façon sur les outils de valorisation à mettre en œuvre pour faire du climatique un nouveau ressort d'attractivité : site patrimonial remarquable, labellisation « Ville d'art et d'histoire » ou «Patrimoine du $\mathrm{xx}^{\mathrm{e}}$ siècle»? Cette stratégie s'inscrit bien entendu dans une volonté de mise en valeur des patrimoines au sens large, notamment naturels, afin de construire un projet de territoire fondé sur les trois piliers du développement durable (environnemental, économique, social) auxquels s'ajoute le quatrième, la culture. Notons qu'en 1945 déjà, le rapport d'Armin Meili faisait de la "protection de la nature " un objectif clé du repositionnement économique des stations suisses: un projet de territoire qui s'appuie sur un site patrimonial remarquable peut inscrire plus facilement les enjeux de protection du patrimoine dans ceux, larges, de la protection de la nature et des sites. La vocation climatique des stations de cure et l'attention portée à leurs spécificités environnementales (exposition, altimétrie, régime hydrique, végétation, etc.) les désigne comme les laboratoires privilégiés d'un développement territorial, articulant patrimoine bâti et qualité environnementale. Au-delà de leurs spécificités, les anciennes stations climatiques de cure sont confrontées aux mêmes problématiques: érosion démographique, baisse de l'activité médicale, concurrence mondialisée en matière de tourisme, incertitudes liées au changement climatique, etc. Il ne fait aucun doute que la mise en réseau de ces collectivités et le partage de leurs expériences complémentaires sera un levier d'adaptation face aux évolutions en cours et leur permettra de mieux structurer une offre économique, sociale et culturelle incluant la mise en valeur des architectures du $\mathrm{xx}^{\mathrm{e}}$ siècle. 
Figure 25

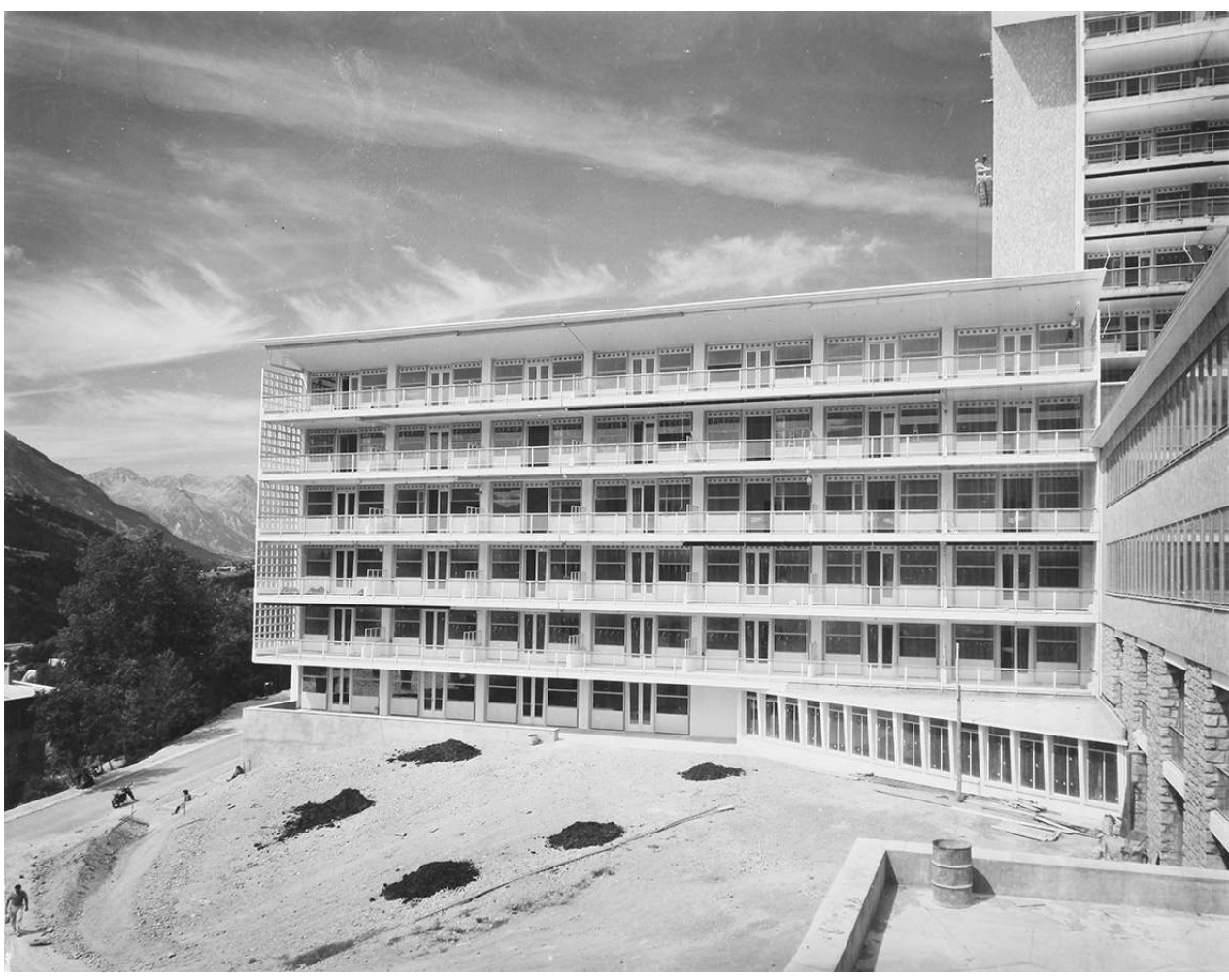

Sanatorium Rhône-Azur (Briançon : Hautes-Alpes, Arati et Boyer architectes, 1957) : façade sud de l'aile des cures. Phot. 1957.

(c) UGECAM PACA.

\section{NOTES}

1. - Le mouvement sanatorial a connu des fortunes diverses en Europe: il fut précoce en Allemagne et en Suisse où l'essentiel de l'effort avait été accompli avant la Première Guerre mondiale, plus tardif en France et en Italie où la construction massive d'établissements de cure fait suite à l'adoption de législations antituberculeuses nationales (en 1919 en France et en 1928 en Italie).

2. - Voir GRANDVOINNET, Philippe. Histoire des sanatoriums en France (1915-1945). Une architecture en quête de rendement thérapeutique. Thèse de doctorat, histoire de l'architecture, sous la direction d'Anne-Marie Châtelet et de Bruno Reichlin. Versailles-Saint-Quentin : universités de VersaillesSaint-Quentin-en-Yvelines et de Genève, 2010. Cette thèse de doctorat a été publiée sous le titre : Architecture thérapeutique: histoire des sanatoriums en France (1900-1945). Genève: MétisPresses, 2014.

3. - SONTAG, Susan. La maladie comme métaphore. Le sida et ses métaphores [1979]. Paris : Christian Bourgois, 1993, p. 24.

4. - REY, Augustin. "Congrès international de la tuberculose ». La Construction moderne, 30 septembre 1905 , p. 115. 
5. - DALLY, Philippe. « Esthétique hospitalière ». L'Architecte, avril 1931, p. 25.

6. - Ibid.

7. - Voir DEL CURTO, Davide. Il sanatorio alpino. Architetture per la cura della tubercolosi dall'Europa alla Valtellina. Rome : Aracne, 2010.

8. - GRANDVOINNET, Philippe. «Le souffle coupé de l'architecture antituberculeuse (1945-1968) ». Dans FARHAT, Georges (dir.). Les années 1960 hic et nunc. Architecture, urbanisme, paysage. Versailles: École nationale supérieure d'architecture/Éditions Recherches, 2010, p. 93-108.

9. - GRANDVOINNET, Philippe. «L'analyse typologique comme outil de sélection : l'exemple des sanatoriums en France ». Dans DROUIN, Martin, RICHARD-BAZIRE, Anne (dir.). La sélection patrimoniale. Montréal : Éditions MultiMondes, coll. «Cahiers de l'Institut du patrimoine de l'UQAM », 2011, p. 43-60.

10. - Voir le site : http://www.zonnestraal.org/ [consulté le 08/11/2016].

11. - JONGE, Wessel de. «Zonnestraal, restauration d'une architecture transitoire ». Dans TOULIER, Bernard, CREMNITZER, Jean-Bernard (dir.). Histoire et réhabilitation des sanatoriums en Europe. Paris : DoCoMoMo International, 2008, p. 62.

12. - Voir : MEURS, Paul, THOOR, Maria Theresia van (dir.). Sanatorium Zonnestraal. History and Restoration of a Modern Monument. Rotterdam : NAi Publishers, 2010.

13. - Voir le site : http://www.alvaraalto.fi/net/paimio/paimio.html [consulté le 08/11/2016].

14. - KAIRANo, Maija. "La rénovation du sanatorium de Paimio». Dans TOULIER, Bernard, CREMNITZER, Jean-Bernard (dir.). Op. cit., p. 71-76.

15. - Les surfaces de béton endommagées ont été dégagées par micro-démolition sur une épaisseur de 3 à $4 \mathrm{~cm}$, suffisante pour mettre en place de nouveaux coffrages. Les profils ont été reformés au moyen de bétons dont les caractéristiques ont été définies après analyse de ceux en place. Les bétons carbonatés, mais non éclatés, ont reçu un simple traitement chimique réalcalinisant empêchant la corrosion des aciers. La pente des balcons a été corrigée de façon à faire rejaillir l'eau en façade et ainsi supprimer les infiltrations récurrentes dans les chambres.

16. - Voir le site : http://swiss-historic-hotels.ch/fr/hotels/cransmontana_bellalui.php [consulté le 08/11/2016]. L'ancien sanatorium Schatzalp, construit à Davos en 1900 et théâtre du roman de Thomas Mann La Montagne magique (1924), est également membre de ce réseau.

17. - Voir le site: http://www.baumundzeit.de [consulté le 08/11/2016]. Les aménagements réalisés dans le cadre de ce projet peuvent être visionnés à ce lien : http://www.baumundzeit.de/ staunen-und-erleben/impressionen.html [consulté le 08/11/2016].

18. - KÖNNINCKE, Peter. «Hoch über den Heilstätten». Potsdamer Neueste Nachichten, 27 septembre 2010.

19. - Les propriétaires de bâtiments protégés au titre de la loi de 1913 sur les monuments historiques peuvent déduire de leur revenu imposable le montant des travaux réalisés sur lesdits édifices.

20. - Voir dans la base Mérimée : notice PA95000005.

21. - Converti en centre de vacances au début des années 1970, le sanatorium de Guébriant n'a pas bénéficié alors de l'attention apte à mettre en valeur ses qualités architecturales et techniques. Repeint dans une teinte ocre rosé proche de celle d'origine, le bâtiment présente aujourd'hui un aspect avenant qui dissimule mal les dégradations qu'il a subies : les menuiseries d'origine ont été remplacées par de larges châssis coulissants en aluminium et la quasi-totalité des aménagements intérieurs a disparu. Cette réalisation unique, sans doute la plus aboutie en matière d'établissements à gradins, avait pourtant fait l'objet de très nombreuses publications en France et à l'étranger dans les années 1930, mais l'opportunité d'une valorisation économique du bâtiment a relégué au second rang le souci de préserver ses caractéristiques architecturales. 
22. - BRUN, Claudine. L'hôpital-sanatorium Sabourin à Clermont-Ferrand. Mémoire de $3^{\mathrm{e}}$ cycle de sauvegarde du patrimoine bâti moderne et contemporain. Genève: Institut d'architecture de l'université de Genève, 2004.

23. - Voir dans la base Mérimée : notice PA63000030.

24. - Ministère de la Culture et de la Communication. Sabourin - Orientations et prescriptions d'ordre patrimonial et historique, novembre 2007.

25. - MEILI, Armin. Bauliche Sanierung von Hotels und Kurorten - Assainissement technique d'hôtels et de stations touristiques. Zürich : Verlag für Architektur Ag. Erlenbach, 1945, p. 10.

26. - Ibid.

27. - Ibid., p. 154-158.

\section{RÉSUMÉS}

Les anciens sanatoriums de cure constituent un élément majeur du patrimoine architectural du $\mathrm{xx}^{\mathrm{e}}$ siècle. Ils incarnent le renouveau d'une architecture hospitalière pour laquelle le malade est devenu l'élément central du projet médical. Les sanatoriums ont ainsi été pendant un demi-siècle un lieu privilégié d'innovation en matière de médecine et d'architecture. Construits pour la plupart entre 1900 et 1950 , ces établissements présentent des dispositions architecturales caractéristiques, propres au traitement de la tuberculose pulmonaire: des bâtiments étirés, éloignés des sources de pollution, si possible en altitude, des parcs conçus pour la promenade, des chambres orientées au sud, des galeries de cure facilement accessibles, des aménagements intérieurs soignés. Après la reconversion massive des années 1960 et 1970, beaucoup de sanatoriums sont aujourd'hui confrontés à la remise en question de leur vocation médicale. Les nombreux projets de reconversion se heurtent à l'enclavement de certains sites de montagne et aux dimensions parfois exceptionnelles des bâtiments. La restauration rigoureuse de quelques éléments emblématiques de ce patrimoine (tels les sanatoriums Zonnestraal aux Pays-Bas, Bella Lui en Suisse ou Paimio en Finlande) ne saurait faire oublier les interventions lourdes qui ont conduit à dénaturer des édifices moins reconnus mais qui présentaient d'indéniables qualités architecturales et paysagères. La réhabilitation d'un ancien sanatorium de cure passe par une analyse exhaustive de ses dispositions architecturales et techniques et par la restitution précise de toutes les modifications intervenues depuis sa mise en service. Ce travail, qui apporte une connaissance approfondie du bâti, précède la mise au point du programme architectural qui devra garantir le maintien de la lisibilité des dispositions d'origine. En montagne, dans les anciennes stations climatiques de cure, la complémentarité des projets touchant plusieurs établissements invite à développer une intervention à échelle urbaine.

Sanatoriums designed for curing diseases such as tuberculosis represent an important component of the architectural heritage of the twentieth century. They are the embodiment of a renewal of hospital architecture where the sick person is the central element of the medical project. For half a century then, these sanatoriums were at the forefront of innovation both in medical and architectural terms. Most of them were constructed between 1900 and 1950 and share some specific architectural features, associated with the treatment of pulmonary tuberculosis. They are generally long buildings, removed from sources of pollution, possibly in the mountains, with surrounding parks designed for health-giving walks, with rooms all facing south, with cure facilities easily accessible and often with high quality finishings. After massive 
modernisation projects during the 1960s and 1970s, many sanatoriums are today confronted with the problems ensuing from the end of their medical functions. Conversion projects are often made difficult by the sanatoriums' isolated location and by their dimensions which are often exceptional. A few remarkable examples of this type of architecture have witnessed exemplary restoration projects (like the Zonnestraal sanatorium in the Netherlands, Bella Lui in Switzerland or Paimio in Finland), but other conversion projects on less well-known sites have been more heavy-handed and have contributed to a loss of architectural and landscape qualities. The proper conversion of a former sanatorium must involve an exhaustive analysis of the building's architectural and technical features as well as a proper understanding of the changes the building has seen since its opening. This preliminary study giving in-depth knowledge of the building should come before the definition of the new functions for the building, in order to guarantee the appreciation of its original dispositions. In mountain regions, where these health facilities were often assembled in climate resorts, the complementarity of the different elements requires a town-plannning approach.

\section{INDEX}

Keywords : architecture, climate heritage, landscape, sanatorium, hospital-sanatorium, climatic health resort, tuberculosis, rehabilitation, restoration, adaptive re-use, upgrading

Mots-clés : architecture, patrimoine climatique, paysage, sanatorium, hôpital-sanatorium, station climatique de cure, tuberculose, réhabilitation, restauration, adaptation, mise aux normes

\section{AUTEUR}

\section{PHILIPPE GRANDVOINNET}

Architecte et urbaniste de l'État, docteur en architecture, membre associé de l'EA 3400 ARCHE philippe.grandvoinnet@culture.gouv.fr 